\title{
Gamma-ray line emission from OB associations and young open clusters
}

\section{The Cygnus region $\star$}

\author{
J. Knödlseder ${ }^{1}$, M. Cerviño ${ }^{2}$, J.-M. Le Duigou ${ }^{1}$, G. Meynet $^{3}$, D. Schaerer ${ }^{4}$, and P. von Ballmoos ${ }^{1}$ \\ 1 Centre d'Étude Spatiale des Rayonnements, CNRS/UPS, BP 4346, 31028 Toulouse Cedex 4, France \\ ${ }^{2}$ LAEFF (INTA) Apdo. 50727, Madrid 28080, Spain \\ 3 Observatoire de Genève, 1290 Sauverny, Switzerland \\ 4 Observatoire Midi-Pyrénées, 14, avenue Édouard Belin, 31400 Toulouse, France
}

Received 22 March 2002 / Accepted 22 May 2002

\begin{abstract}
Gamma-ray and microwave observations of the Cygnus region reveal an intense signal of $1.809 \mathrm{MeV}$ line emission, attributed to radioactive decay of ${ }^{26} \mathrm{Al}$, that is closely correlated with $53 \mathrm{GHz}$ free-free emission, originating from the ionised interstellar medium. We modelled both emissions using a multi-wavelength evolutionary synthesis code for massive star associations that we applied to the known massive star populations in Cygnus. For all OB associations and young open clusters in the field, we determined the population age, distance, and richness as well as the uncertainties in all these quantities from published photometric and spectroscopic data. We propagate the population uncertainties in model uncertainties by means of a Bayesian method. The young globular cluster $\mathrm{Cyg} \mathrm{OB} 2$ turns out to be the dominant ${ }^{26} \mathrm{Al}$ nucleosynthesis and ionisation source in Cygnus. Our model reproduces the ionising luminosity of the Cygnus region very well, yet it underestimates ${ }^{26} \mathrm{Al}$ production by about a factor of 2 . We attribute this underestimation to shortcomings of current nucleosynthesis models, and suggest the inclusion of stellar rotation as possible mechanism to enhance ${ }^{26} \mathrm{Al}$ production. We also modelled ${ }^{60} \mathrm{Fe}$ nucleosynthesis in the Cygnus region, yet the small number of recent supernova events suggests only little ${ }^{60} \mathrm{Fe}$ production. Consequently, a detection of the $1.137 \mathrm{MeV}$ and $1.332 \mathrm{MeV}$ decay lines of ${ }^{60} \mathrm{Fe}$ from Cygnus by the upcoming INTEGRAL observatory is not expected.
\end{abstract}

Key words. nuclear reactions, nucleosynthesis, abundances - open clusters and associations: general

\section{Introduction}

$\mathrm{OB}$ associations and young open clusters constitute the most prolific nucleosynthesis sites in our Galaxy. The combined activity of stellar winds and core-collapse supernovae ejects significant amounts of freshly synthesised nuclei into the interstellar medium. Radioactive isotopes, such as ${ }^{26} \mathrm{Al}$ or ${ }^{60} \mathrm{Fe}$, that have been co-produced in such events, may eventually be observed by gamma-ray instruments through their characteristic decay-line signatures. Indeed, galactic $1.809 \mathrm{MeV}$ gamma-ray line emission attributed to the radioactive decay of ${ }^{26} \mathrm{Al}$ has been observed by numerous gamma-ray telescopes (see Prantzos \& Diehl 1996 for a review). In particular, the COMPTEL telescope provided the first image of the Galaxy in the light of this isotope, showing an asymmetric ridge of diffuse emission along the galactic plane with a prominent localised emission enhancement in the Cygnus region (Diehl et al. 1995).

Send offprint requests to: J. Knödlseder,

e-mail: knodlseder@cesr.fr

* Appendices A and B, and Tables 1,2, and 5 are only available in electronic form at http://www . edpsciences.org
The Cygnus emission has been interpreted as the result of ${ }^{26} \mathrm{Al}$ ejection in Wolf-Rayet winds and during core collapse supernova explosions from a nearby $(1-2 \mathrm{kpc})$ massive star population which probably is part of the local spiral arm structure (del Rio et al. 1996).

Gamma-ray line emission is not the only tracer of this activity. Knödlseder et al. (1999a) demonstrated that the galactic $1.809 \mathrm{MeV}$ emission is closely correlated to galactic freefree emission as observed in the microwave domain. The free-free emission mainly results from the ionisation of the interstellar medium by the UV flux of O stars, hence it traces the massive star population. The observed correlation is indeed one of the strongest arguments in favour of prolific ${ }^{26} \mathrm{Al}$ production by massive stars. In general, a wealth of distinct massive star populations of different ages, sizes, or metallicities contribute to the emission along a line of sight through the Galaxy, and the observed correlation allows only conclusions about the average properties of the contributing populations. In that way, the observations suggest that the equivalent $\mathrm{O} 7 \mathrm{~V}$ star ${ }^{26} \mathrm{Al}$ yield, defined as the average amount of ${ }^{26} \mathrm{Al}$ ejected per number of $\mathrm{O} 7 \mathrm{~V}$ star (measured by their equivalent ionising production), has a 
galaxywide constant value of $Y_{26}^{\mathrm{O} 7 \mathrm{~V}}=(1.0 \pm 0.3) \times 10^{-4} M_{\odot}$ (Knödlseder 1999).

It is surprising, however, that the correlation between $1.809 \mathrm{MeV}$ and microwave free-free emission also holds for the Cygnus region. Both gamma-ray and microwave data show a localised emission enhancement towards Cygnus, similar in size and relative intensity, resulting in an equivalent O7V star ${ }^{26} \mathrm{Al}$ yield of $(1.1 \pm 0.3) \times 10^{-4} M_{\odot}$ (see Sect. 4). Within the uncertainties this yield is identical to the galactic value. In contrast to the Galaxy, however, only few massive star associations contribute to the observed emission in Cygnus, and it is not expected that they have the same properties as the Galaxy as a whole. In particular, the galactic metallicity gradient leads to an average galactic abundance that is supersolar, and indeed only a supersolar abundance is able to reconcile theoretical ${ }^{26} \mathrm{Al}$ yields with the observed galactic ${ }^{26} \mathrm{Al}$ mass (Knödlseder 1999). In contrast, massive star populations in Cygnus show slightly subsolar abundances (e.g. Daflon et al. 2001) and since ${ }^{26} \mathrm{Al}$ yields are believed to depend on metallicity (e.g. Prantzos \& Diehl 1996) the nucleosynthetic properties of the Cygnus region should deviate from those of the average Galaxy.

To understand the observations, we present in this paper a bottom-up model of the Cygnus region where we aim to explain the gamma-ray and microwave data from the underlying stellar populations. For this purpose we developed a multiwavelength evolutionary synthesis model that we presented in Paper I of this series (Cerviño et al. 2000). We put considerable effort into the characterisation of the massive star populations in Cygnus with particular emphasis on the involved uncertainties (distance and age uncertainty; coeval or continuous star formation). We incorporate these uncertainties into our model by means of a Bayesian method and determine confidence intervals for all quantities to assess the predictive power of our approach. Despite the resulting uncertainties, we will demonstrate that the gamma-ray observations provide important clues on nucleosynthesis physics in massive stars. In particular we will demonstrate the shortcomings of current theoretical nucleosynthesis models in explaining ${ }^{26} \mathrm{Al}$ production and discuss possible modifications that may improve the models.

\section{Evolutionary synthesis model and analysis method}

The evolutionary synthesis model we employed in this work is described in detail in the first paper of this series by Cerviño et al. (2000). In summary, the evolution of each individual star in a stellar population is followed using Geneva evolutionary tracks with enhanced mass-loss rates (Meynet et al. 1994). Stellar Lyman continuum (Lyc) luminosities are predicted using the CoStar atmosphere models of Schaerer \& de Koter (1997), supplemented by the Schmutz et al. (1992) atmospheres for the Wolf-Rayet phase. At the end of stellar evolution, stars initially more massive than $M_{\mathrm{WR}}=25 M_{\odot}$ are exploded as Type Ib supernovae, while stars of initial mass within $8 M_{\odot}$ and $M_{\mathrm{WR}}$ are assumed to explode as Type II SNe. Nucleosynthesis yields have been taken from Meynet et al. (1997) for the pre-supernova evolution, from Woosley \& Weaver (1995) for Type II and from Woosley et al. (1995) for
Type Ib supernova explosions. Note that Type II SN yields have only been published for stars without mass loss and Type $\mathrm{Ib}$ yields have only been calculated for pure Helium stars. In order to obtain consistent nucleosynthesis yields for Type II supernovae we followed the suggestion of Maeder (1992) and linked the explosive nucleosynthesis models of Woosley \& Weaver (1995) to the Geneva tracks via the carbon-oxygen core mass at the beginning of carbon burning. For Type Ib SN we used the helium-core mass at the beginning of helium core burning for the link.

In order to predict the present gamma-ray line emission from a massive star association we have to estimate the production of radioactive isotopes in the past. We do this by determining the actual number of stars $N_{*}$ within a given initial mass interval $\left[M_{\text {low }}, M_{\text {up }}\right]$ that is not affected by incompleteness at the lower end and evolutionary effects at the upper end. We then extrapolate this population back to the past using a Salpeter initial mass function (IMF) of slope $\Gamma=-1.35$ that is normalised to the number of stars we observe today. In practice, we generate an initial stellar population by randomly sampling the IMF until the number of stars falling in the mass interval $\left[M_{\text {low }}, M_{\text {up }}\right]$ amounts to $N_{*}$. We then use the evolutionary synthesis code to follow the time evolution of the ${ }^{26} \mathrm{Al}$ and ${ }^{60} \mathrm{Fe}$ yields, the Lyc luminosity, and the distribution of spectral types within the population.

The stellar systems we study in this work only comprise a few hundred to a few thousand stars, making the high-mass end of the population generally sparsely sampled. However, massive stars provide a considerable fraction of the association nucleosynthesis and ionising power, and the early evolution of the system will depend rather sensitively on the actual choice of stellar masses. In particular, different Monte Carlo samples of the same initial stellar population may lead to quite different evolutions of the association observables, leading to considerable uncertainties in our model predictions. We decided to include these uncertainties in our analysis by means of a Bayesian method. Instead of investigating the time evolution of a quantity $X$ for a specific population, we base our analysis on the probability density function (PDF) $p(X \mid t)$ which quantifies the probability for $X$ to be equal to a value $x$ at the time $t$. We approximate this function for a given association by repeating the evolutionary synthesis calculations for independently sampled stellar populations, from which we obtain the frequency of observing the value $x$ at time $t$. In this way, the uncertainty arising from the extrapolation to the past, which manifests in an uncertainty about the initial massive star population, is reflected in the width of the PDF. Examples of PDFs obtained for massive star associations in the Cygnus regions are given in Cerviño et al. (2000).

To predict the today value of the quantity $X$, we marginalise over the (uncertain) age $t$ of the association using

$p(X)=\int_{0}^{\infty} p(t) p(X \mid t) \mathrm{d} t$

where the prior probability distribution $p(t)$ quantifies the age uncertainty. We tried different choices for the prior distributions, such as Gaussians or bounded uniform functions, but we found that the precise form of the prior has little impact on 
the result as long as it quantifies our knowledge about the age uncertainty (see Sect. 4.2.1). To estimate fluxes $S_{i}$ from luminosities $L_{i}$ for a given association $i$, we further marginalise over the distance $s$ of the association using

$p_{i}\left(S_{i}\right)=\int_{0}^{\infty} p(s) p\left(L_{i} \mid s\right) \mathrm{d} s$,

where the prior $p(s)$ quantifies the distance uncertainty, and $S_{i} \propto s^{-2}$. Integral fluxes from the Cygnus region were obtained by adding the contributions from individual OB associations and young open clusters using successive marginalisation, i.e.

$p(S)=\int_{0}^{\infty} p_{1}\left(S_{1}\right) p_{2}\left(S-S_{1}\right) \mathrm{d} S_{1}$,

where $p_{1}$ and $p_{2}$ are the flux PDFs for association 1 and 2, respectively, and $S=S_{1}+S_{2}$. Finally, we derive mean (or median) flux values and $63.8 \%$ confidence regions from these PDFs which we then compare to observations.

\section{Massive star populations in Cygnus}

The starting point of our study of massive star populations in Cygnus is the WEBDA database of open clusters of Mermilliod (1998). From this database we established a list of open clusters within the limits $60^{\circ}<l<110^{\circ}$ and $|b|<15^{\circ}$, a region which largely encloses the $1.809 \mathrm{MeV}$ and microwave free-free emission features. We preselected young clusters from this list by requiring either an earliest spectral type of $\mathrm{B} 3 \mathrm{~V}$ or earlier (corresponding to a star of $\sim 8 M_{\odot}$ initial mass), or a cluster age of less than $5 \times 10^{7} \mathrm{yr}$. Older clusters do not house any potential core collapse progenitor anymore, and their nucleosynthesis activity is therefore negligible. The lack of stars more massive than $\sim 10 M_{\odot}$ also reduces their ionising flux to an imperceptible level. Of course, not for all known open clusters an age estimate is available, but those clusters are then often rather distant and/or do not contain luminous members. Consequently, they do not contribute significantly to the emissions that we aim to study in this work.

For the $\mathrm{OB}$ associations in the Cygnus region we gathered the relevant information from the literature. Our main resources were the compilations of Humphreys (1978) and Garmany \& Stencel (1992), complemented with data from the SIMBAD database and some recent investigations (see the appendix for a detailed description of our database).

The association parameters relevant to our study are the age $\tau$, the distance $d$, the richness, expressed as the number of stars $N_{*}$ in a specific initial mass interval [ $\left.M_{\text {low }}, M_{\text {up }}\right]$, and the slope $\Gamma$ of the IMF (in the following we deliberately choose the term association when we talk both about $\mathrm{OB}$ associations and young open clusters). Literature values for these parameters are only available for some of the associations, and have generally been derived by different methods. Also the choice of the stellar evolutionary tracks and luminosity calibrations impacts the results, and may lead to differences among the derived parameters. Additionally, the uncertainty of the association parameters has rarely been determined, and if so, also not in a consistent manner. For these reasons we decided to re-determine the relevant association parameters and their uncertainties from the basic stellar data in our database. In the following we describe our method and present the resulting parameters for all young stellar associations in Cygnus. The results of the analysis are summarised in Tables 1 and 2. Details for each association are given in the appendix.

\subsection{Reddening}

As a first step we determine the reddening for each association from spectroscopic and photometric information. For each star with spectral classification we used the spectral type to intrinsic colour calibration of FitzGerald (1970) complemented by information from Schmidt-Kaler (1982) for the earliest spectral types to determine their colour excess $E(B-V)=(B-$ $V)-(B-V)_{0}$ and $E(U-B)=(U-B)-(U-B)_{0}$. From these we calculate the slope $q_{\mathrm{r}}=E(U-B) / E(B-V)$ of the reddening curve for each association by averaging the slopes of all member stars with known spectral type. Outliers were iteratively removed from the average until all remaining stars lie within 2 rms of the average.

The slopes typically vary from 0.67 to 0.84 with a few exceptions with poorly defined values due to the proximity of the association (Cyg OB7, Lac OB1, Roslund 5). We find a trend towards steeper slopes (typically around $q_{\mathrm{r}}=0.80$ ) for the more reddened associations, in particular in the area of Cyg OB1, OB2 and OB9, i.e. the central part of the Cygnus $\mathrm{X}$ region. This confirms findings by other authors (e.g. Massey \& Thompson 1991) of an anomalous reddening in this area. For the couple of open clusters for which no spectral data are available, the canonical value of $q_{\mathrm{r}}=0.72$ has been adopted. In view of the dispersion in the value of $q_{\mathrm{r}}$ in the Cygnus area this choice is certainly somewhat arbitrary, but the impact of the exact value on the resulting cluster parameters is rather small, leading to a negligible additional uncertainty in our study.

\subsection{Distance}

As a second step the distance of each association is estimated. For associations with spectroscopic information we employed the method of spectroscopic parallax. For each MK classified star we compute $D M=V-A_{\mathrm{V}}-M_{\mathrm{V}}$, where $A_{\mathrm{V}}=R_{\mathrm{V}} \times E(B-V)$ is the visible extinction. The association distance is then calculated by averaging $D M$ where again $2 \mathrm{rms}$ outliers were removed iteratively. The absolute visual magnitudes $M_{\mathrm{V}}$ have been extracted from calibrations of Vacca et al. (1996) for stars earlier than B1, complemented by data from Humphreys \& McElroy (1984) and Schmidt-Kaler (1982) for later spectral types. For the ratio $R_{\mathrm{V}}$ of absolute-to-selective absorption we apply the canonical value of 3.1 , although we recognise that deviations from this value are eventually observed in star forming regions (Mathis 1990). For Cyg OB2, for example, Massey \& Thompson (1991) found $R_{\mathrm{V}}=3.0$, and indeed a variable extinction analysis of our data suggests a similar value. This deviation results in a distance modulus error of 0.2 mag for this association, inferior to the statistical and systematical uncertainty of $0.5 \mathrm{mag}$. Cyg OB2 is certainly an extreme case due to the large reddening of the association, and we believe 
that variations in $R_{\mathrm{V}}$ should not dramatically alter our results. Anyways, we have little choice since generally the available data do not allow to perform a reliable variable extinction analysis for the associations, and we fear that a poorly determined value of $R_{\mathrm{V}}$ introduces a larger error than a rather solid mean value of 3.1 that may not precisely apply to all cases.

For some of the open clusters for which not enough spectral type information was available, we estimated the distance from the hot cluster stars using the reddening-free parameter $Q=(U-B)-q_{\mathrm{r}} \times(B-V)$, where $q_{\mathrm{r}}$ is the mean cluster reddening slope as given in Table 1 . For $Q<-0.4$, corresponding to spectral types earlier than B5 or so, there exist unique relations between intrinsic colour $(B-V)_{0}$, absolute visual magnitude $M_{\mathrm{V}}$, and $Q$ for each luminosity class. We determined these relations by fitting polynomial functions to calibration tables that we compiled from FitzGerald (1970), Schmidt-Kaler (1982), Humphreys \& McElroy (1984), and Vacca et al. (1996). To estimate the luminosity class we apply an iterative approach. For each star with $Q<-0.4$ we assume as initial estimate a luminosity class of $\mathrm{V}$ (this is certainly a reasonable assumption for young unevolved clusters). Based on this estimate we use the calibration relations to determine $(B-V)_{0}$ and $M_{\mathrm{V}}$ from $Q$. From these quantities we derive $E(B-$ $V), A_{\mathrm{V}}$, and $D M$, leading to a first cluster distance estimate by averaging over $D M$. Cluster stars which we misclassified in luminosity class will clearly show up as outliers in $D M$ with respect to the average. Hence, for stars with $D M$ more than $1 \mathrm{rms}$ above the average we reduce the luminosity class (in the sense $\mathrm{V} \rightarrow \mathrm{I})$ while for stars with $D M$ more than $1 \mathrm{rms}$ below the average we increase the luminosity class (in the sense $\mathrm{I} \rightarrow \mathrm{V}$ ) for the next iteration. We then repeat the entire procedure for the updated luminosity classes. For all clusters in our sample, this scheme led to convergence in $D M$ after 2-3 iterations. We tested the validity of our procedure by applying the method to clusters for which $D M$ is known from spectroscopic parallaxes and found satisfactory agreement with the photometric results.

\subsection{Field star separation}

For Cyg OB2 and many of the clusters, field star contamination is a serious problem in our database. Field stars may be recognised by particularly low or high $E(B-V)$ values with respect to bona fide association members (e.g. Massey et al. 1995). For contaminated associations, we therefore excluded those stars whose inferred colour excess is outside the range spanned by member stars with MK classification. Most clusters show a clear upper main sequence which allows for an unambiguous definition of stellar reddenings of cluster members. For the cases where we applied such a selection, the $E(B-V)$ range of cluster members is specified in Col. 5 of Table 1.

To perform such a selection, $E(B-V)$ has been determined for each star from either spectroscopic or photometric data. In the latter case, the iterative procedure described above has been used for hot stars with $Q<-0.4$. For $Q \geq-0.4$, the relation between $(B-V)_{0}$ and $Q$ becomes ambiguous, corresponding to the well known "knee" in the colour-colour diagram. Nevertheless, from the intersection of the reddening lines with the intrinsic colour-colour tracks we can determine all possible $E(B-V)$ values (3 at maximum) that are consistent with the observed colours, and we exclude all stars that have no solution that is compatible with the specified colour excess interval.

To remove remaining field or background stars we employed for most clusters another selection criterion based on the distance moduli of the stars. From bona fide members with MK classification we define a distance moduli interval $\triangle D M$ and exclude all stars for which $|D M-\langle D M\rangle|>\Delta D M$, where $\langle D M\rangle$ is the distance modulus of the association. Distance moduli for stars with MK classification or hot $(Q<-0.4)$ stars were determined from spectroscopic and photometric parallaxes, respectively. For the remaining stars, distance moduli were again estimated from the intersection of the reddening lines with the intrinsic colour-colour tracks.

\subsection{Transformation to $\log T_{\text {eff }}$ and $M_{\text {bol }}$}

Our next step consists of constructing H-R diagrams (HRDs) for all associations. For this purpose we transform the spectroscopic and photometric information for all member stars to $\log T_{\text {eff }}$ and $M_{\text {bol }}$. If a star has a MK classification, we used the spectral type to determine both the effective temperature and bolometric correction B.C. using the calibration of Vacca et al. (1996), complemented by data from Humphreys \& McElroy (1984) and Schmidt-Kaler (1982). From the absolute visual magnitude and the bolometric correction, the bolometric luminosity is then derived using $M_{\mathrm{bol}}=M_{\mathrm{V}}+B . C$.

For stars without MK classification, we again distinguish between hot $(Q<-0.4)$ and cold $(Q \geq-0.4)$ stars. For hot stars we determined $\log T_{\text {eff }}$ and B.C. from $Q$, while for cold stars we derived both parameters from the intrinsic colour $(B-V)_{0}$. Using the mean association distance $D M$ and extinction $A_{\mathrm{V}}$ we assign a bolometric luminosity to each star using $M_{\mathrm{bol}}=V-A_{\mathrm{V}}-D M+B . C$. We estimate the intrinsic colour $(B-V)_{0}=(B-V)-E(B-V)$ for each star by assuming a reddening identical to the mean reddening of the association. Our analysis is based on polynomial functions that we fitted to the calibration tables of intrinsic stellar parameters. In general, the relations depend of the luminosity class which we estimate for each star from the absolute visual magnitude $M_{\mathrm{V}}=V-A_{\mathrm{V}}-D M$ (unless it has already been estimated by the iterative procedure described in Sect. 3.2). Here we make use of the rapid variation of $M_{\mathrm{V}}$ with luminosity class, and we search the calibration tables for the luminosity class that comes closets to the pair $M_{\mathrm{V}}$ and $(B-V)_{0}$ of stellar parameters. Although this method is certainly not very accurate, the impact of a misclassification in luminosity class is only moderate. Typically, $\log T_{\text {eff }}$ varies about $0.02-0.1 \mathrm{dex}$ and B.C. about $0.3-0.7 \mathrm{mag}$ between subsequent luminosity classes.

\subsection{Age determination}

From the H-R diagrams we estimated the age of the associations. For this purpose we compared the location of the association stars in the HRDs to the theoretical evolutionary tracks of Meynet et al. (1994) for solar metallicity $(Z=0.02)$. The same 


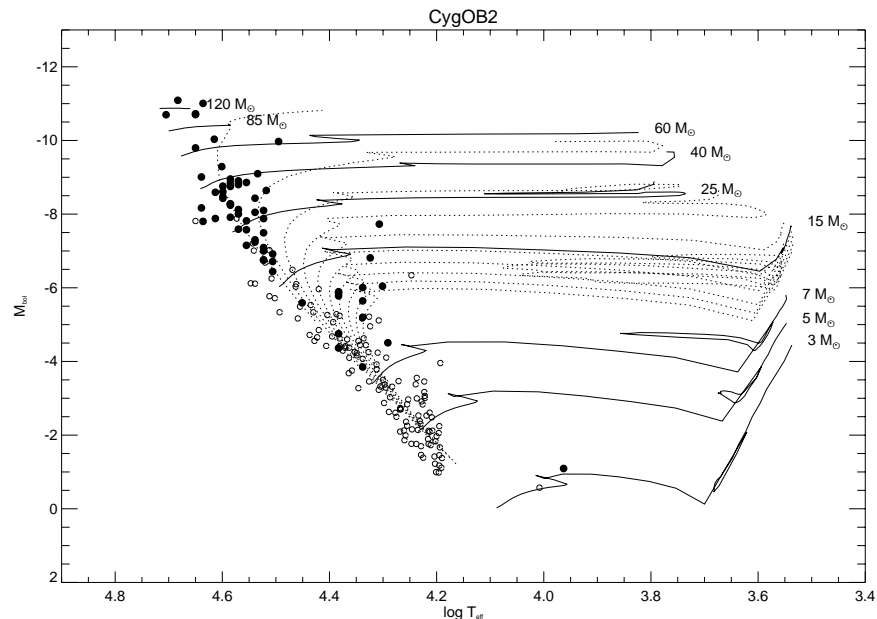

Fig. 1. H-R diagram of Cyg OB2. The filled circles are stars for which spectral classification is available, the open circles are stars for which we have only photometry. Solid lines are the stellar tracks of Meynet et al. (1994), dotted lines are the corresponding isochrones for intervals of $2 \mathrm{Myr}$, starting from $\tau=2 \mathrm{Myr}$.

tracks are used for the evolutionary synthesis calculations, hence in this respect our models are consistent with the population input data. As an example we show the HRD for Cyg OB2 in Fig. 1 which may be compared to Fig. 16 of Massey \& Thompson (1991) who made a similar analysis. From the upper part of the HRD (masses $\gtrsim 20 M_{\odot}$ ) we infer the ages of the massive stars from the Meynet et al. (1994) isochrones that we superimposed on the diagrams. For Cyg OB2, for example, this method leads us to an age estimate of 1-4 Myr, comparable to the finding of Herrero et al. (1999).

The results for all associations are summarised in Table 2. Instead of determining a best fitting age, we always tried to specify an age range that reflects the dispersion of the upper main sequence. The dispersion comes partly from the systematic limitation of our method (knowledge of stellar calibrations, neglection of stellar rotation and binarity, measurement or classification errors), but could also reflect a period of continuous star formation. In particular, some associations like Cep OB1, Cyg OB8 or Roslund 4 show a considerable scatter in the upper HRD which could even reflect several epochs of massive star formation. For Cep OB1 we decided to split the population into two subgroups of different age since the data are difficult to reconcile with a single star formation event. Yet, a large apparent age spread could also signal that the association presents not a physically associated group but rather a chance projection of massive stars in the sky. For this reason we excluded Cyg OB4 from our study since Hipparcos data did not confirm the reality of this stellar group (de Zeeuw et al. 1999). Other associations such as Cyg OB8 or Vul OB1 are also highly doubtful, yet it will turn out that they provide only a negligible contribution to the overall luminosities in the Cygnus region, hence we do not introduce an important uncertainty by including them into our study.
Table 3. IMF slopes $\Gamma$ for two OB associations and some open clusters. The third column indicates the mass range over which the slope has been determined.

\begin{tabular}{lcc}
\hline \hline Name & $\Gamma$ & mass range $\left(M_{\odot}\right)$ \\
\hline Cyg OB1 & $-1.0 \pm 0.4$ & $15-85$ \\
Cyg OB2 & $-1.1 \pm 0.3$ & $15-120$ \\
\hline Berkeley 86 & $-1.2 \pm 0.5$ & $7-40$ \\
Berkeley 87 & $-1.2 \pm 0.4$ & $7-60$ \\
IC 4996 & $-1.6 \pm 0.5$ & $5-40$ \\
NGC 6823 & $-1.3 \pm 0.4$ & $7-60$ \\
NGC 6871 & $-1.0 \pm 0.5$ & $7-40$ \\
NGC 6910 & $-0.9 \pm 0.4$ & $5-40$ \\
NGC 6913 & $-0.6 \pm 0.5$ & $7-60$ \\
NGC 7235 & $-2.6 \pm 0.6$ & $7-40$ \\
NGC 7380 & $-0.9 \pm 0.5$ & $7-40$ \\
Trumpler 37 & $-1.1 \pm 0.4$ & $7-60$ \\
\hline
\end{tabular}

\subsection{Association richness and IMF}

The comparison of the H-R diagram with the evolutionary tracks allows an estimation of the initial richness of the association. We determined the richness by counting the number of stars $N_{*}$ over a sufficiently large initial mass interval $\left[M_{\text {low }}, M_{\text {up }}\right]$. We tried to choose the lower mass limit $M_{\text {low }}$ sufficiently high to avoid any bias from population incompleteness due to the limiting magnitudes of the association surveys. Conversely, the upper mass limits $M_{\text {up }}$ were chosen sufficiently low so that the evolutionary turn-off has no impact on the richness determination. The results are summarised in Cols. 8 and 9 of Table 2.

The only exception to this procedure is Cyg OB2 for which we rely on the recent mass determination of Knödlseder (2000), based on an analysis of $2 M A S S$ near infrared data. In fact, the data that are available in the visible waveband for Cyg OB2 are heavily affected by interstellar absorption, and their use would considerably underestimate the total mass of this association. In contrast, the $2 M A S S$ data do not allow a precise spectral type determination and the quoted number of $120 \mathrm{O}$ stars rather reflects the fact that there were initially 120 stars more massive than $\sim 20 M_{\odot}$ in Cyg OB2, although some may already have evolved, having either turned in early B-type or even WolfRayet stars. The quoted number of $120 \mathrm{O}$ stars should therefore present an upper limit.

For some associations our stellar database contains enough objects to allow an estimation of the initial mass spectrum. We fitted these spectra by power-law initial mass functions and summarise their slopes $\Gamma$ in Table 3 . We also quote the mass intervals that have been used for the fitting which again were chosen to minimise any bias due to evolved stars, population incompleteness, and field star contamination. Yet we recognise that the results are still affected by systematic uncertainties, as demonstrated by the steep slope of $\Gamma=-2.6 \pm 0.6$ for NGC 7235 which shows a heavy field star contamination (Massey et al. 1995). Formally, we derive a weighted mean IMF slope of $\Gamma=-1.2 \pm 0.1$ which is close to the canonical Salpeter value of -1.35 and compatible with the value of 
$\Gamma=-1.12 \pm 0.08$ that has been determined by Massey et al. (1995) for 12 galactic OB associations and open clusters. To illustrate the systematic uncertainties in IMF slope determinations, we compare our result for Cyg OB2 $(-1.1 \pm 0.3)$ to the value of $-1.0 \pm 0.1$ determined by Massey \& Thompson (1991) from the same stellar data, and the recent analysis of Knödlseder (2000) of 2MASS near infrared data that suggests $\Gamma=-1.6 \pm 0.1$. In view of these uncertainties, we believe that the assumption of a uniform canonical Salpeter law for our evolutionary synthesis modelling of all associations is a reasonable approximation that is compatible with the observational data.

\subsection{The Cygnus superbubble}

The discovery of an extended X-ray ring-like source surrounding the Cygnus $\mathrm{X}$ region by Cash et al. (1980) has been interpreted as a superbubble blown by the massive star winds and supernova explosions in the Cyg OB2 association. Anomalous stellar proper motions have been reported for stars in associations located near the edge of the bubble (Comerón et al. 1993), and have been interpreted as the sign for triggered star formation following gravitational instability of the expanding shell (Comerón \& Torra 1994). However, the energetic requirements needed to explain the expansion motion are difficult to reconcile with the mechanical power provided by Cyg OB2 (Comerón et al. 1998). In addition, the superbubble is equally well, if not even better, explained as a superposition of multiple objects at different distances that aline along the local spiral arm that is seen tangentially in this direction (Bochkarev \& Sitnik 1985; Uyaniker et al. 2001).

Also our cluster analysis seems difficult to reconcile with the scenario of an expanding bubble that triggered formation of the associations surrounding Cyg OB2. Firstly, the central association Cyg OB2 is one of the youngest in the area (1-4 Myr) while the surrounding associations Cyg OB1, OB3, OB7, and OB9 are slightly older (2-6 Myr). In the scenario where Cyg OB2 triggered the formation of the other associations, however, the situation should be vice versa. Secondly, only Cyg OB1 and OB3 are situated at distances that are compatible with that of $\mathrm{Cyg}$ OB2 while Cyg OB7 and OB9 are likely foreground objects. In addition, Cyg OB2 is not situated at the centre of the X-ray superbubble and the other OB associations correlate only partly with the structure (Uyaniker et al. 2001), making a physical relation of these objects highly questionable. Thirdly, the expansion age determined by Comerón et al. (1998) from stellar proper motions fits surprisingly well our age estimate for Cyg OB2, supporting the idea that the expanding stars are dynamically ejected runaway stars from Cyg OB2 (Comerón et al. 1998). Comerón et al.'s list of candidate members of the Cygnus expanding structure contains four O-type stars, which compared to the total of 120 O-type stars in Cyg OB2 (Knödlseder 2000) would result in a runaway star fraction of $3 \%$ for this association. Compared to other young massive star associations, such as the Trapezium cluster in Orion or the $\lambda$ Orionis star-forming region (Hoogerwerf et al. 2001), such a proportion seems typical, and we believe that at least part, if not all, of the anomalous stellar proper motions in Cygnus are indeed explained by runaway stars.

\subsection{First estimates}

Using simple approximations we can already make a first order estimate of the ionising flux that we expect from the stellar populations in the Cygnus region. The CoStar models of Schaerer $\&$ de Koter (1997) predict a Lyman continuum luminosity of $\log Q_{0}=49.05 \mathrm{ph} \mathrm{s}^{-1}$ for a star of spectral type O7V, and assuming that all $204 \mathrm{O}$ stars that we find in the associations of the Cygnus region are indeed O7V stars, we derive a total luminosity of $\log Q=51.36 \mathrm{ph} \mathrm{s}^{-1}$. Assuming further that all stars lie at a typical distance of $1.8 \mathrm{kpc}$, and using the relation

$S_{53}(\mathrm{Jy})=8.06 \times 10^{-48} \frac{Q}{s^{2}}\left(\frac{T_{\mathrm{e}}}{8000 \mathrm{~K}}\right)^{-0.45}$

( $s$ is the distance in units of kpc, $Q$ is the Lyc luminosity given in $\mathrm{ph} \mathrm{s}^{-1}$, and $T_{\mathrm{e}}$ is the electron temperature which we assume to $8000 \mathrm{~K}$; cf. Afflerbach et al. 1997) we predict a $53 \mathrm{GHz}$ freefree emission flux of $\sim 5700 \mathrm{Jy}$. We will see in the next section that this flux level is of the same order as the observed value.

\section{Modelling the Cygnus region}

\subsection{Gamma-ray and microwave observations}

Before we present the results of our evolutionary synthesis modelling we want to discuss the gamma-ray and microwave observations that triggered our investigation. Maps of 1.809 MeV gamma-ray line emission and $53 \mathrm{GHz}$ free-free emission of the Cygnus regions are shown in superposition in Fig. 2. The spatial correlation between both emission features is striking. However, we want to caution the reader of overinterpretations of the apparent structures in the map. The gamma-ray feature has a total significance of only $\sim 8 \sigma$, and the detailed morphology of the image is heavily biased by instrumental noise (Knödlseder et al. 1999b). Formally, the COMPTEL instrument had an angular resolution of $\sim 4^{\circ}$ $(F W H M)$, yet the small amplitude of the signal probably does not allow inferences at angular scales below $\sim 10^{\circ}$. This is comparable to the angular resolution of $7^{\circ}(F W H M)$ for the $C O B E$ DMR experiment which was used to derive the free-free emission map.

Observations of galactic radio-continuum emission from Cygnus at smaller angular scales indicate that most of the emission originates from a region between $l \sim 74^{\circ}$ and $84^{\circ}$ with emission peaks around $l \sim 80^{\circ}$ (e.g. Wendker 1970). This region coincides with the OB associations Cyg OB1, Cyg OB2, Cyg OB8, and Cyg OB9 and a large number of the youngest open clusters in our database. However, the small-scale radio maps that are available were generally obtained at lower frequencies where synchrotron emission contributes significantly to the signal. Although the Cygnus emission is primarily optically thin thermal radiation, a superimposed smooth non-thermal component makes the determination of absolute free-free emission levels difficult (Wendker et al. 1991). Despite the poor angular resolution, we therefore prefer using 

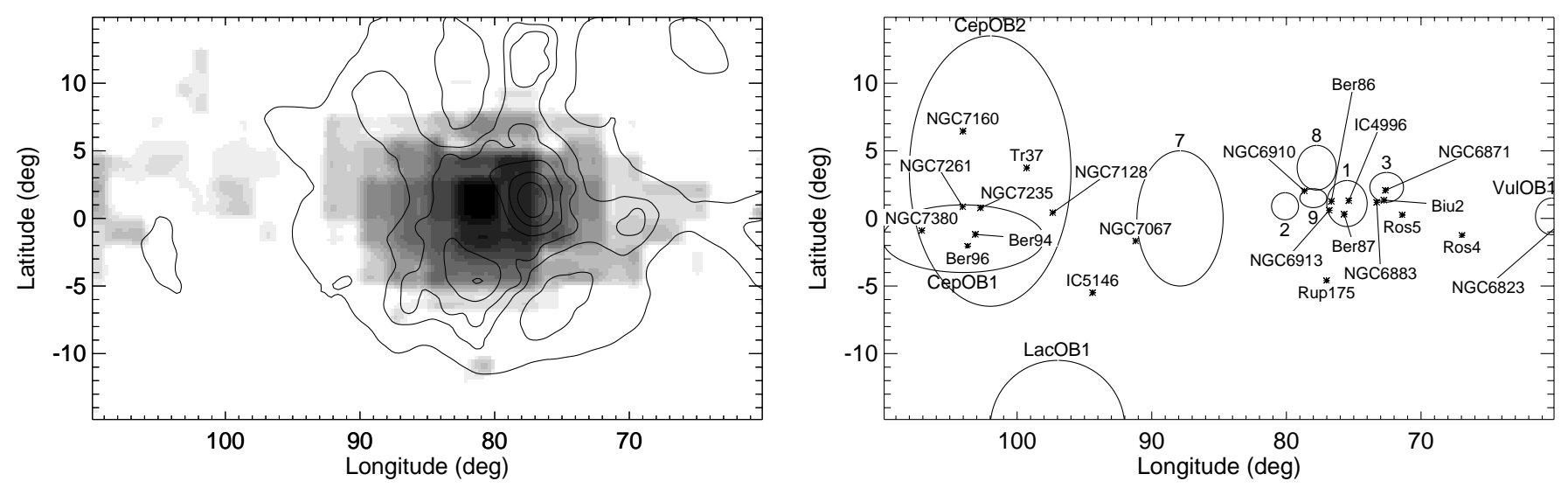

Fig. 2. Left: contour map of $1.809 \mathrm{MeV}$ gamma-ray line emission in the Cygnus region (Plüschke 2001) superimposed onto a greyscale image of microwave $53 \mathrm{GHz}$ free-free emission (Bennett et al. 1992). The prominent emission feature extending from $l \sim 70^{\circ}$ to $90^{\circ}$ and $b \sim \pm 8^{\circ}$ is known as Cygnus $\mathrm{X}$ region. Right: finding chart for OB associations (circles) and young open clusters (asterisks) in the Cygnus region. For Cyg OB1 to Cyg OB9 only the OB numbers are quoted.

the $53 \mathrm{GHz}$ free-free emission map of Bennett et al. (1992) for the estimation of the ionising flux in Cygnus. At this frequency the contribution from synchrotron and thermal dust emission is small within the galactic plane, and can easily be removed by modelling their spatial distribution at adjacent frequencies. The image shown in Fig. 2 results from such a separation performed on DMR data (Bennett et al. 1992).

Smaller angular scales are probably even not desirable for a global flux comparison, although they bear valuable information about the interplay between the massive stars with the interstellar medium. Firstly, at angular scales that are smaller than the associations, the flux distribution may be influenced by individual massive stars, which we do not attempt to model in our study. Secondly, the correlation between free-free emission and the massive star distribution may anyway break-down at scales comparable to the association dimensions due to the lack of gas in their ploughed interiors. For example, Huchtmeier \& Wendker (1977) did not find any $\mathrm{H}$ II region inside Cyg OB2 despite the large number of ionising stars that is present. They argue that the association is devoid of hydrogen and the ionising flux is converted into free-free emission by the surrounding gas, leading to a diffuse extended emission component. Thirdly, the long lifetime of ${ }^{26} \mathrm{Al}(\tau \sim 1 \mathrm{Myr}$ ) allows the isotope to travel substantial distances before the emission of the gammaray decay photons, and the detailed $1.809 \mathrm{MeV}$ emission structure should depend considerably on the interaction and deceleration of the ejecta in the surrounding interstellar medium. Morphology studies that address the question of ejecta propagation in the Cygnus region will become possible with the upcoming INTEGRAL observatory (e.g. Knödlseder \& Vedrenne 2001), but the existing COMPTEL data limits our analysis to only a global investigation.

We determined the $1.809 \mathrm{MeV}$ and $53 \mathrm{GHz}$ free-free fluxes from the Cygnus region by integrating the respective skymaps over the area of interest. A diffuse galactic ridge emission that underlies the features in the Cygnus region has been subtracted from the flux estimates in order to extract the emission that is correlated to the Cygnus associations. The resulting flux estimates are only weakly sensitive on the precise location of
Table 4. Flux measurements and derived equivalent $\mathrm{O} 7 \mathrm{~V}$ star ${ }^{26} \mathrm{Al}$ yield of the Cygnus region. For comparison, the galactic average $Y_{26}^{\mathrm{O} 7 \mathrm{~V}}$ value is also quoted.

\begin{tabular}{ll}
\hline \hline Quantity & Measured value \\
\hline$S_{1809}$ & $(5.8 \pm 1.5) \times 10^{-5} \mathrm{ph} \mathrm{cm}^{-2} \mathrm{~s}^{-1}$ \\
$S_{1137}$ & - \\
$S_{53}$ & $4200 \pm 700 \mathrm{Jy}$ \\
$Y_{26}^{\text {O7V }}$ & $(1.1 \pm 0.3) \times 10^{-4} M_{\odot}$ \\
$Y_{26}^{\text {O7V }}($ Galaxy $)$ & $(1.0 \pm 0.3) \times 10^{-4} M_{\odot}$ \\
\hline
\end{tabular}

the integration boundaries. To safely cover the investigated region we selected a longitude interval from $l=65^{\circ}$ to $110^{\circ}$ for the flux determination and determined the galactic ridge background from two adjacent intervals at $l=110^{\circ}-130^{\circ}$ and $l=60^{\circ}-65^{\circ}$. We selected a rather large latitude window of $|b|<30^{\circ}$ to assure that we recover also the tails of the emission (in particular the COMPTEL skymap shows extended high latitude wings due to the low significance of the reconstructed emission). The resulting $1.809 \mathrm{MeV}$ gamma-ray line flux amounts to $S_{1809}=(5.8 \pm 1.5) \times 10^{-5} \mathrm{ph} \mathrm{cm}^{-2} \mathrm{~s}^{-1}$ while the $53 \mathrm{GHz}$ free-free emission flux equals $S_{53}=4200 \pm 700 \mathrm{Jy}$. The quoted errors reflect both the statistical and the systematical uncertainties in the flux determination, in particular those introduced by the selection of the integration boundaries.

The flux measurements can be converted into the equivalent $\mathrm{O} 7 \mathrm{~V}$ star ${ }^{26} \mathrm{Al}$ yield using

$Y_{26}^{\mathrm{O} 7 \mathrm{~V}}\left(M_{\odot}\right)=7.91 \times 10^{3} \frac{S_{1809}\left(\mathrm{ph} \mathrm{cm}^{-2} \mathrm{~s}^{-1}\right)}{S_{53}(\mathrm{Jy})}$.

We obtain $Y_{26}^{\mathrm{O} 7 \mathrm{~V}}=(1.1 \pm 0.3) \times 10^{-4} M_{\odot}$ for the Cygnus region, a value that is compatible with the galactic average value of $(1.0 \pm 0.3) \times 10^{-4} M_{\odot}$. The flux measurements are summarised together with the equivalent ${ }^{26} \mathrm{Al}$ yields in Table 4 . 


\subsection{Evolutionary synthesis modelling}

\subsubsection{Prior probabilities and model uncertainties}

We used the method described in Cerviño et al. (2000) and Sect. 2 to estimate the nucleosynthesis yields and Lyc luminosity for each association. The results are compiled in Table 5. For each association 100 statistically independent evolutionary synthesis models have been calculated to approximate the PDFs for all quantities of interest. Based on our age determinations, we marginalised the age uncertainty using Eq. (1) with bounded Gaussian-shaped prior probability distributions. The mean and standard deviation of the Gaussians were defined by the mean and the half-width of the age boundaries quoted in Col. 2 of Table 2; the prior density was set to zero for ages outside the age boundaries. The ${ }^{26} \mathrm{Al}$ and ${ }^{60} \mathrm{Fe}$ yields as well as the Lyc luminosities that result from this procedure are given in Cols. 2-4 of Table 5.

Alternatively, we also tried box-shaped prior distributions that were bounded by our age estimates, but the results were only slightly different. In fact, Eq. (1) performs a weighted average of the PDFs where the age prior $p(t)$ defines the weights that are attributed to each age. From our association analysis we derive a possible range of ages, yet the data are not sufficiently accurate to derive details about the age distribution. Therefore, any prior distribution that reflects reasonably well the fact that the association age is comprised between two limits should provide a valid prior for our analysis. The only difference between the Gaussian and the box-shaped prior is that the first slightly favours models with an age close to the mean age between the boundaries, while the second gives equal weight to all ages that are compatible with the assigned limits. Since the observed age spread is at least partly due to measurement uncertainties, and since we defined our age boundaries as two extreme limits that enclose the age distribution of the most luminous stars, we feel that a bounded Gaussian-shaped prior reflects most properly the information that we have at hand about the association ages.

We also want to emphasise that our age marginalisation is equivalent to the assumption of a continuous star formation over an interval defined by the age boundaries. In this interpretation, the form of the age prior specifies the time evolution of the star formation rate. Since this rate is generally unknown, a rather flat prior distribution, like those we discussed above, is certainly appropriate. The interpretation of the apparent age distribution in the H-R diagram is usually a difficult enterprise due to the blending between measurement uncertainties, spectral misclassification, neglection of binarity and rotation, nonmember pollution, and a possible non-coeval star formation. We cannot distinguish between all these uncertainties either, yet our formalism includes all of them into the model results.

The nucleosynthesis yields and ionising luminosity were converted into gamma-ray line and microwave free-free fluxes by marginalising the distance uncertainty for each association (Eq. (2)). For the $1.809 \mathrm{MeV}$ gamma-ray line flux due to the radioactive decay of ${ }^{26} \mathrm{Al}$ we used the relation

$S_{1809}\left(\mathrm{ph} \mathrm{cm}^{-2} \mathrm{~s}^{-1}\right)=1.18 \times 10^{-2} \frac{Y_{26}}{s^{2}}$

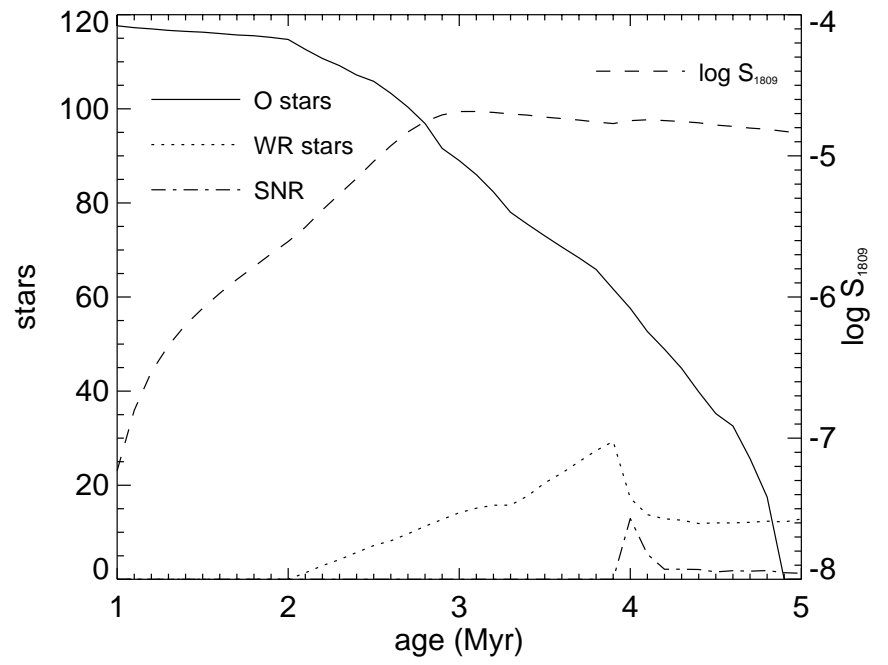

Fig. 3. Evolution of the number of O stars, Wolf-Rayet stars, supernova remnants, and $1.809 \mathrm{MeV}$ gamma-ray line flux as function of age for Cyg OB2. We assumed a lifetime of $10^{5} \mathrm{yr}$ for each SNR.

while for the $1.137 \mathrm{MeV}$ gamma-ray line flux from the decay of ${ }^{60} \mathrm{Fe}$ we employed

$S_{1137}\left(\mathrm{ph} \mathrm{cm}^{-2} \mathrm{~s}^{-1}\right)=2.41 \times 10^{-3} \frac{Y_{60}}{s^{2}}$

( $Y_{26}$ and $Y_{60}$ are the ${ }^{26} \mathrm{Al}$ and ${ }^{60} \mathrm{Fe}$ yields in units of $M_{\odot}$, respectively, $s$ is the distance in units of $\mathrm{kpc}$ ). Note that the ${ }^{60} \mathrm{Fe}$ decay leads in fact to two gamma-ray lines at 1.137 and $1.332 \mathrm{MeV}$, but since their branching ratio and thus their intensity is basically identical we only quote the flux for the $1.137 \mathrm{MeV}$ line. The conversion from ionising luminosity to $53 \mathrm{GHz}$ freefree flux is given by Eq. (4). As distance priors $p(s)$ we used Gaussians with means and standard deviations that were defined by the distance moduli means and errors given in Col. 3 of Table 1. Again, since the distance modulus uncertainty arises primarily from measurement uncertainties, we believe that a Gaussian-shaped prior is a reasonable representation of our distance knowledge. The resulting $1.809 \mathrm{MeV}, 1.137 \mathrm{MeV}$, and $53 \mathrm{GHz}$ fluxes are quoted in Cols. 5-7 of Table 5.

Our evolutionary synthesis model also predicts the spectral type distribution, and we quote in Cols. 8-9 of Table 5 the predicted number of O-type and Wolf-Rayet stars. These predictions can be directly compared to the respective columns of Table 2, and we globally find a good agreement although the median values often fall below the observed values. The exception to the rule is Cyg OB2 for which the models predict between 51 and $116 \mathrm{O}$ stars while the observations suggest 120. However, as discussed in Sect. 3.6, the $120 \mathrm{O}$ stars quoted in Table 2 are rather an upper limit since the 2MASS analysis did not account for possible evolutionary effects. Yet, Cyg OB2 houses evolved stars and even shows indications for non-coeval and ongoing star formation (e.g. Torres-Dodgen et al. 1991; Massey \& Thompson 1991; Parthasarathy et al. 1992; Pigulski \& Kolaczkowski 1998; Herrero et al. 1999).

The effect of the age uncertainty on the stellar population in Cyg OB2 is illustrated in Fig. 3. For a coeval star formation event, the number of $\mathrm{O}$ stars drops rapidly from the initial 


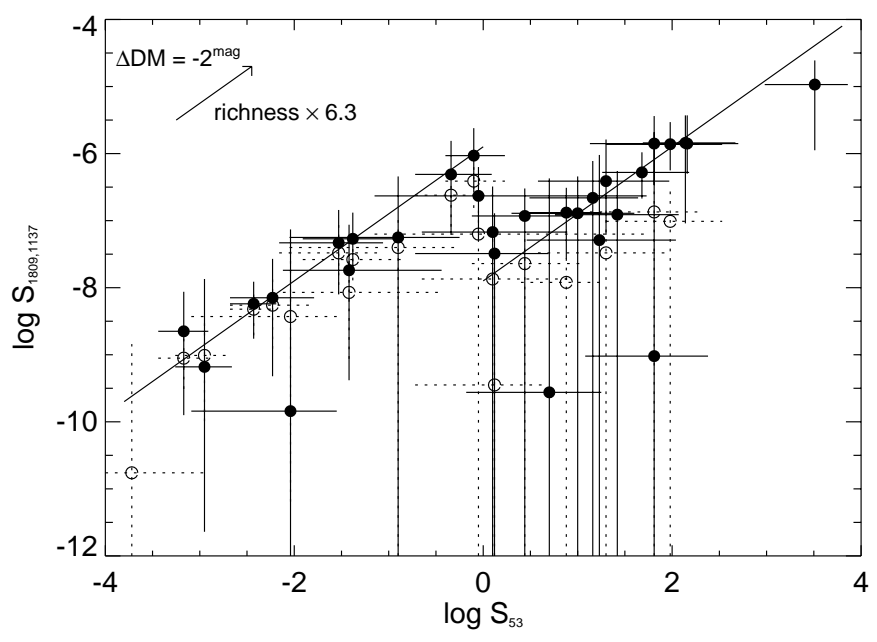

Fig. 4. Predicted $1.809 \mathrm{MeV}$ (filled dots and solid error bars) and 1.137 MeV gamma-ray line fluxes (open dots and dashed error bars) as function of predicted $53 \mathrm{GHz}$ free-free flux. The arrow indicates the displacement due to a decrease of $2 \mathrm{mag}$ in the distance modulus or an increase of the richness by a factor of 6.3. The two solid lines indicate equivalent ${ }^{26} \mathrm{Al}$ O7V star yields of $\log Y_{26}^{\mathrm{O} 7 \mathrm{~V}}=-2$ (left) and -4 (right).

value of 120 to zero during the first $5 \mathrm{Myr}$ of the association evolution. Clearly, there is a considerable number of O-type stars in Cyg OB2 (e.g. Massey et al. 1991), hence the associations should be younger than 5 Myr. Wolf-Rayet stars appear around 2 Myr after the starburst while the first supernovae explode about 4 Myr after the birth of the association. The fact that there are Wolf-Rayet stars associated with Cyg OB2 suggests an age $\gtrsim 2 \mathrm{Myr}$, the presence of 2 WC-type stars even indicates an age above $3 \mathrm{Myr}$. On the other hand no supernova remnant has been detected within the boundaries of Cyg OB2 (Wendker et al. 1991), hence the association should be younger than $\sim 4$ Myr. The $1.809 \mathrm{MeV}$ gamma-ray line flux raises from zero to a maximum level of $2 \times 10^{-5} \mathrm{ph} \mathrm{cm}^{-2} \mathrm{~s}^{-1}$ within $\sim 3 \mathrm{Myr}$ (for an assumed distance modulus of $D M=11.0 \mathrm{mag}$ ), and Cyg OB2 could be situated just at the maximum of the $1.809 \mathrm{MeV}$ gamma-ray light curve, which is about a factor of two above the median value quoted in Table 5. Nevertheless, the present reasoning only holds for a strictly coeval star formation event, and the evidence for ongoing star formation in Cyg OB2 holds against narrowing down the age boundaries for the association. Clearly, more observational data is required to improve our knowledge about the age distribution in Cyg OB2 before we safely can improve upon the nucleosynthesis yield estimation for this association.

This example illustrates that the apparent age spread in the HRDs presents an important source of uncertainty for the final flux estimates, at least for the youngest associations. In general, however, the dominating source of uncertainty depends on the association richness and its age, but also on the nature of the quantity of interest. For Cyg OB2, for example, the $53 \mathrm{GHz}$ flux prediction is much less affected by the age uncertainty than the $1.809 \mathrm{MeV}$ flux estimate. While the $1.809 \mathrm{MeV}$ flux varies by more than two magnitudes between 1-4 Myr, the number of $\mathrm{O}$ stars changes only by a factor of two, implying a $53 \mathrm{GHz}$ flux variation by a factor of less than six. For slightly older associations, such as Trumpler 37, the situation is reversed: the $1.809 \mathrm{MeV}$ flux varies only slowly while the ionising flux now drops quickly due to the disappearing of the most massive stars in supernovae events.

If the association is sufficiently rich so that sampling effects are negligible, the distance uncertainty turns out to be the dominating factor of the flux dispersion. Typically, the relative distance uncertainty for an association amounts to $20 \%$ which directly translates into a flux uncertainty of $40 \%$. Sampling effects are important when only few stars contribute to the association luminosities, i.e. either for very young or for very poor populations. Cyg OB7 fulfils both conditions. For this association, a considerable fraction of the evolutionary synthesis samples did not lead to any ${ }^{26} \mathrm{Al}$ production at all. In those cases, the initial mass function has not been populated by sufficiently massive stars to lead to a noticeable ${ }^{26} \mathrm{Al}$ production during the conceivable age range of Cyg OB7 (2-5 Myr). We indicate such a possibility by quoting infinite negative errors in Table 5.

\subsubsection{Correlated emission}

To investigate the correlation between gamma-ray line flux and free-free emission in the Cygnus region we plotted the predicted 1.809 and $1.137 \mathrm{MeV}$ fluxes versus the $53 \mathrm{GHz}$ flux (Fig. 4). All three quantities should scale with distance and association richness, and indeed we find a general trend of increasing gamma-ray intensity with increasing free-free flux. A few associations with low gamma-ray fluxes $\left(\log S_{1809,1137} \approx-9\right)$ deviate from this tendency, yet they represent those poorly populated clusters that show a tremendous flux dispersion due to poor sampling of the high-mass IMF (see above). To illustrate the distance and richness impact, we superimposed an arrow in Fig. 4 that indicates the displacement due to a decrease of 2 mag in the distance modulus or an equivalent increase of the association richness by a factor of 6.3 .

For the $1.809 \mathrm{MeV}$ flux, the associations are situated along this line of displacement, yet there occurs a clear discontinuity around $\log S_{53} \approx 0$. All associations with $\log S_{53}<0$ are older than $10 \mathrm{Myr}$, an evolutionary phase during which ${ }^{26} \mathrm{Al}$ is exclusively ejected by supernovae events (the only exception to this rule are Berkeley 94 and 96, yet they are so sparsely populated that no ${ }^{26} \mathrm{Al}$ production is expected). In contrast, all associations with $\log S_{53}>0$ are younger than $10 \mathrm{Myr}$, hence they are dominated by ${ }^{26} \mathrm{Al}$ ejection through stellar winds (Cerviño et al. 2000). The discontinuity in Fig. 4 represents a change in the equivalent $\mathrm{O} 7 \mathrm{~V}$ star ${ }^{26} \mathrm{Al}$ yield, and we find typical values of $\log Y_{26}^{\mathrm{O} 7 \mathrm{~V}} \approx-2$ and -4 for $\log S_{53}<0$ and $>0$, respectively.

The discontinuity in the correlation coefficient may eventually lead to a break-down in the tight correlation between $1.809 \mathrm{MeV}$ and $53 \mathrm{GHz}$ emission, which should occur however at rather low $1.809 \mathrm{MeV}$ flux levels of about $10^{-6} \mathrm{ph} \mathrm{cm}^{-2} \mathrm{~s}^{-1}$. At this level, it is expected to find $1.809 \mathrm{MeV}$ emission features that are uncorrelated to microwave free-free emission (or for which the free-free emission should not exceed an intensity of $1 \mathrm{Jy}$ ). Given the sensitivity limit of the spectrometer SPI on INTEGRAL, it is unlikely that this break-down will be observable in the near future. 

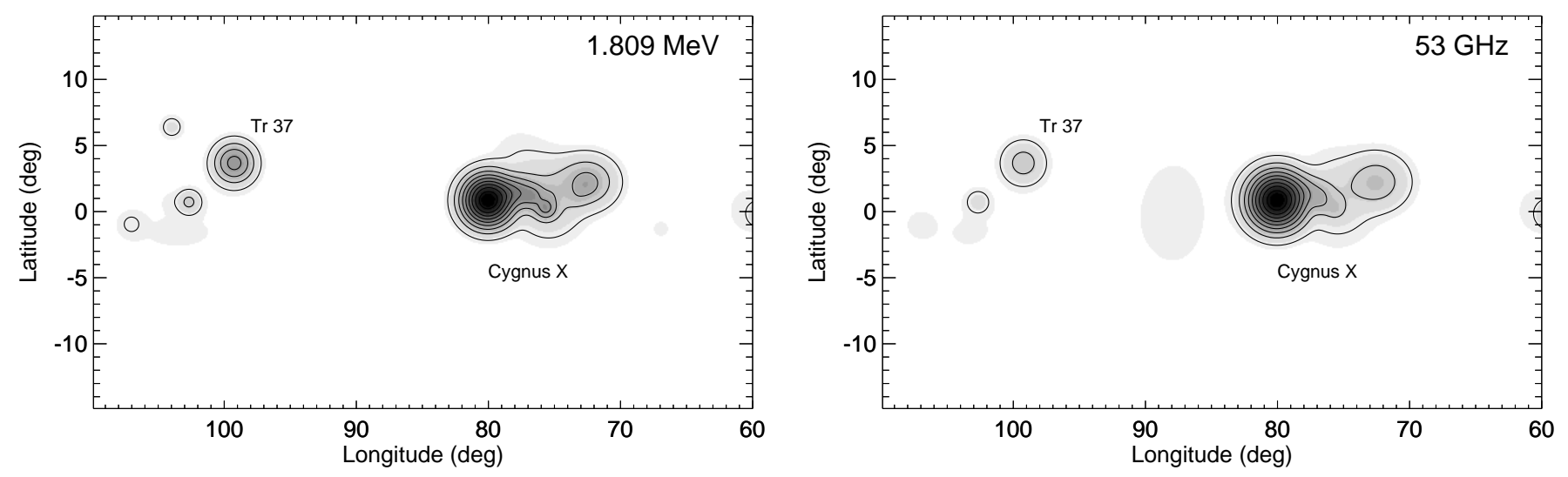

Fig. 5. Predicted median $1.809 \mathrm{MeV}$ gamma-ray line intensity distribution (left) and $53 \mathrm{GHz}$ microwave free-free intensity (right).

For the $1.137 \mathrm{MeV}$ flux we find only a weak correlation with free-free emission, which is mainly limited to $\log S_{53}<0$, i.e. older associations that are dominated by supernova explosions. For those, a typical equivalent $\mathrm{O} 7 \mathrm{~V}$ star ${ }^{60} \mathrm{Fe}$ yield of $\log Y_{60}^{\mathrm{O} 7 \mathrm{~V}} \approx-1.5$ is found, yet the low level of the associated free-free flux $\left(S_{53}<1 \mathrm{Jy}\right)$ will inhibit the observation of this correlation. For $\log S_{53}>0,1.137 \mathrm{MeV}$ fluxes are highly uncertain due to the small number of supernova events that contribute to ${ }^{60} \mathrm{Fe}$ production (recall that no large amounts of wind-ejected ${ }^{60} \mathrm{Fe}$ is expected from massive stars; see Cerviño et al. 2000). The youngest associations, such as Cyg OB2 or Cyg OB3, show no ${ }^{60} \mathrm{Fe}$ production at all, despite the huge amounts of ionising photons that is produced by their large number of O-type stars.

By far the most luminous association in both $1.809 \mathrm{MeV}$ and $53 \mathrm{GHz}$ free-free emission is Cyg OB2, which corresponds to the isolated dot in the upper-right corner of Fig. 4. The median equivalent yield of Cyg OB2 amounts to $Y_{26}^{\mathrm{O} 7 \mathrm{~V}}=$ $2.2 \times 10^{-5} M_{\odot}$, somewhat lower than the observed value of $1.1 \times 10^{-4} M_{\odot}$ in the Cygnus region. In other words, Cyg OB2 seems to underproduce ${ }^{26} \mathrm{Al}$ with respect to ionising photons, which is mainly related to its extremely young age. Most of the massive stars are still on the main sequence where they produce large amounts of ionising photons, but do not yet eject copious amounts of ${ }^{26} \mathrm{Al}$ in the interstellar medium.

For the entire Cygnus region, we find an equivalent O7V star ${ }^{26} \mathrm{Al}$ yield of $Y_{26}^{\mathrm{O} 7 \mathrm{~V}}=4.7 \times 10^{-5} M_{\odot}$, about a factor of two below the observed value. Apparently, our model underpredicts ${ }^{26} \mathrm{Al}$ production with respect to the ionising luminosity by about a factor of 2 , and we will suggest later that this underprediction is most likely related to shortcomings of current nucleosynthesis models for massive stars. It is interesting to note that our predicted $Y_{26}^{\mathrm{O} 7 \mathrm{~V}}$ value is very close to the steadystate value of $4.9 \times 10^{-5} M_{\odot}$, which results for a stellar population with a constant star formation rate (Cerviño et al. 2000). Since the Cygnus region is largely dominated by a single association, Cyg OB2, we believe that this finding is merely a coincidence. Indeed, our predicted equivalent $\mathrm{O} 7 \mathrm{~V}$ star ${ }^{60} \mathrm{Fe}$ yield of $Y_{60}^{\mathrm{O} 7 \mathrm{~V}}=2.0 \times 10^{-5} M_{\odot}$ for the entire Cygnus region falls more than a factor of two below the steady-state value of $5.6 \times 10^{-5} M_{\odot}$ (Cerviño et al. 2000), emphasising the relative rareness of supernova events in the Cygnus region with respect to a steady-state population.

\subsubsection{Spatial distribution}

To illustrate the spatial distribution of the $1.809 \mathrm{MeV}$ and $53 \mathrm{GHz}$ emission predicted by our model, we show in Fig. 5 intensity maps for both observables that are based on the median flux values quoted in Table 5. Although we have no information about the spatial distribution of the respective emission within and around the associations, we distributed the flux within the association boundaries using Gaussian-shaped density profiles to account for the spatial extent of the stellar populations. We recall that the true distribution could be more widespread due to the possible lack of ionised gas in the ploughed cluster interiors and the propagation of the radioactive ejecta before decay (cf. Sect. 4.1). In addition, when comparing these distributions to the observed maps in Fig. 2, the angular resolution of the telescopes of $4^{\circ}$ (COMPTEL) and $7^{\circ}$ (DMR) has to be considered.

Taking these factors into account, the morphological resemblance of our model maps to the observations is quite reasonable. We predict free-free and $1.809 \mathrm{MeV}$ emission maxima at $(l, b) \sim\left(80^{\circ}, 1^{\circ}\right)$ which spatially coincides with the observed maximum of the $53 \mathrm{GHz}$ map, and is also close the maxima in the $1.809 \mathrm{MeV}$ COMPTEL map. The observations show copious emission in the longitude range $l \sim 70^{\circ}-90^{\circ}$, and also our model predicts the bulk emission in this region. Obviously, the most prolific sources of $1.809 \mathrm{MeV}$ and $53 \mathrm{GHz}$ emission are (in the quoted order) the OB associations Cyg OB2, Cyg OB3, Cyg OB9, and Cyg OB1 that form the core of the Cygnus $\mathrm{X}$ complex (see Fig. 2). Cyg OB2 is by far the most important source in the area for which we predict median fluxes of $S_{1809}=1 \times 10^{-5} \mathrm{ph} \mathrm{cm}^{-2} \mathrm{~s}^{-1}$ and $S_{53}=3200 \mathrm{Jy}$. Comparison of the median values with the observed fluxes from the entire region (cf. Sect. 4.1) suggests that Cyg OB2 alone could provide around $75 \%$ of the ionisation in Cygnus while it may account for only $\sim 20 \%$ of the observed $1.809 \mathrm{MeV}$ photons. Including both observational and modelling uncertainties weakens this discrepancy, yet the general trend remains: within the uncertainties, Cyg OB2 could easily explain 
all ionisation in Cygnus while it can account at most for $60 \%$ of the observed ${ }^{26} \mathrm{Al}$.

Using successive marginalisation (Eq. (3)) we estimate the total flux from the associations and clusters in the Cygnus $\mathrm{X}$ area $\left(70^{\circ} \leq l \leq 82^{\circ} ;-3^{\circ} \leq b \leq 6^{\circ}\right)$ to $S_{1809}=\left(2.0_{-1.1}^{+1.6}\right) \times$ $10^{-5} \mathrm{ph} \mathrm{cm}^{-2} \mathrm{~s}^{-1}, S_{1137}=\left(3.4_{-2.6}^{+6.1}\right) \times 10^{-8} \mathrm{ph} \mathrm{cm}^{-2} \mathrm{~s}^{-1}$, and $S_{53}=4000_{-2300}^{+3700} \mathrm{Jy}$. The ionising flux is in comfortable agreement with the integrated $53 \mathrm{GHz}$ emission from the Cygnus region, while the $1.809 \mathrm{MeV}$ flux falls significantly below the observed value. The predicted $1.137 \mathrm{MeV}$ flux is way below the detection limit of any envisagable gamma-ray telescope, reflecting the extreme rareness of supernova events in this area. Stellar-wind ejection seems to dominate the enrichment of the interstellar medium by nucleosynthesis products in the Cygnus $\mathrm{X}$ region - more than $85 \%$ of the $1.809 \mathrm{MeV}$ flux in our model originates from ${ }^{26} \mathrm{Al}$ that is ejected through this channel.

The second feature in the model map is low-level $1.809 \mathrm{MeV}$ and $53 \mathrm{GHz}$ free-free emission from some open clusters in the Cepheus region, in particular from Trumpler 37. Trumpler 37 is a young, rich, and nearby open cluster that is embedded into the bright $\mathrm{H}_{\text {II }}$ region IC 1396. Distance estimates based on Hipparcos data vary between $D M=9.1$ and 9.9 mag (de Zeeuw et al. 1999; Robichon et al. 1999) while our analysis results in a value of $10.2 \pm 0.4$ mag. Using this larger estimate we predict a median $1.809 \mathrm{MeV}$ flux of $1.6 \times 10^{-6} \mathrm{ph} \mathrm{cm}^{-2} \mathrm{~s}^{-1}$. Adopting the short Hipparcos distance of de Zeeuw et al. (1999) would result in a flux of $S_{1809}=3.9 \times 10^{-6} \mathrm{ph} \mathrm{cm}^{-2} \mathrm{~s}^{-1}$, making Trumpler 37 a potential source of $1.809 \mathrm{MeV} \gamma$-ray line emission in an area that is little affected by source confusion. COMPTEL observations (cf. Fig. 2) do not show any feature in this area, yet the predicted flux is considerably below the sensitivity limit of this instrument. With its improved sensitivity, the SPI telescope aboard the upcoming INTEGRAL observatory may be able to detect this emission, in particular if current nucleosynthesis models indeed underestimate ${ }^{26} \mathrm{Al}$ production in massive stars (see below).

\subsubsection{Integrated emission}

Finally, the luminosities of all associations have been combined by successive marginalisation (Eq. (3)) to predict the total fluxes from the Cygnus region; the results are presented in the last row of Table 5. For the entire Cygnus region we obtain a median $53 \mathrm{GHz}$ flux of $5000 \mathrm{Jy}$, close to the observed value of $4200 \pm 700 \mathrm{Jy}$. The total predicted $1.809 \mathrm{MeV}$ flux, however, is significantly below the observations: while the model predicts a median gamma-ray line flux of $S_{1809}=$ $2.5 \times 10^{-5} \mathrm{ph} \mathrm{cm}^{-2} \mathrm{~s}^{-1}$ and a $63.8 \%$ confidence interval of (1.6-4.0) $\times 10^{-5} \mathrm{ph} \mathrm{cm}^{-2} \mathrm{~s}^{-1}$, the COMPTEL data suggest a value of $(5.8 \pm 1.5) \times 10^{-5} \mathrm{ph} \mathrm{cm}^{-2} \mathrm{~s}^{-1}$. Our model predicts only little ${ }^{60} \mathrm{Fe}$ synthesis in Cygnus, and consequently, the predicted median $1.137 \mathrm{MeV}$ flux amounts to only $S_{1137}=$ $2 \times 10^{-6} \mathrm{ph} \mathrm{cm}^{-2} \mathrm{~s}^{-1}$. At this level, it seems unlikely that the SPI telescope will detect the radioactive decay of the ${ }^{60} \mathrm{Fe}$ isotope in the Cygnus region.
On average, about $80 \%$ of the $1.809 \mathrm{MeV}$ emission in our model originates from ${ }^{26} \mathrm{Al}$ ejected by stellar winds while only $20 \%$ come from core-collapse supernovae. Also, OB associations play a dominant role in the Cygnus region since they contribute to almost $80 \%$ of the $1.809 \mathrm{MeV}$ emission and to about $90 \%$ of the free-free luminosity.

Our model predicts 99-172 O stars and 13-34 Wolf-Rayet stars in the Cygnus region associations, about $\sim 25 \%$ less than we find in our cluster database. We think that this discrepancy is acceptable, in particular in view of the strong variation of the $\mathrm{O}$ star number with association age (Fig. 3). Note that in any case, the missing $\mathrm{O}$ stars cannot explain the apparent underestimation of the $1.809 \mathrm{MeV}$ luminosity, since lowering the age estimates of some associations (in particular Cyg OB2) to increase the number of $\mathrm{O}$ stars tends also to lower the ${ }^{26} \mathrm{Al}$ production. On the other hand, an important increase in the number of O stars will also increase proportionally the ionising flux for the Cygnus region, leading quickly to an overproduction of Lyc photons.

\section{Discussion}

One essential finding from a census of all known clusters and OB associations and our modelling effort is that we can comfortably reproduce the ionising flux from the Cygnus region, while we fall short by a factor of 2 in explaining ${ }^{26} \mathrm{Al}$ nucleosynthesis. There are two possibilities that may lead to such a result: either the nucleosynthesis models we employed underpredict ${ }^{26} \mathrm{Al}$ production by a about a factor of 2 , or we overpredict the ionising flux by about a factor of 2 , and in the same time, underestimate the stellar population in the Cygnus region by about the same factor. We tend to believe that the problem is more likely related to the nucleosynthesis models, and in the following we will present the elements that lead us to this conclusion.

\subsection{Ionisation}

Our prediction of the ionising flux from the Cygnus region relies on stellar atmosphere models that predict the stellar Lyman continuum luminosities as function of the stellar parameters. However, the lack of spectroscopic data in the extreme ultraviolet shortward of the hydrogen ionisation limit leaves these models basically untested in this wavelength region, and we have to consider the reliability of the employed atmosphere models in predicting Lyc fluxes.

The status of predictions of ionising fluxes has recently been reviewed by Schaerer (1998, 1999). From a comparison of Lyman continuum photon fluxes predicted by various atmospheric models, Vacca et al. (1996) concluded that they are consistent to within 20\%. Oey \& Kennicutt (1997) compared observed $\mathrm{H}$ II region luminosities in the Large Magellanic Cloud to the Lyman continuum luminosities predicted from their well-determined stellar content, using the CoStar atmosphere models of Schaerer \& de Koter (1997) that we also employed in this work. They find that on average, $\mathrm{H}$ II region luminosities amount to $74 \%$ of the ionising luminosities predicted by the stellar models, leaving room for about $25 \%$ flux 
overprediction by the models. Hunter \& Massey (1990) come to a similar conclusion, using the stellar Lyc estimates of Panagia (1973) applied to small galactic $\mathrm{H}$ II regions. However, accounting for the downward revision of the effective temperature scale due to non-LTE line blanketing effects, reduces the Lyc fluxes for a given spectral type (Martins et al. 2002) eliminating the overprediction found by the above studies.

On the other hand, it is commonly believed that some fraction of the ionising photons escape the $\mathrm{H}$ II regions and ionise the diffuse, warm, ionised medium (WIM) of the Galaxy (e.g. Oey \& Kennicutt 1998). The WIM has been found to comprise $20-53 \%$ of the total $\mathrm{H} \alpha$ luminosity in nearby star-forming galaxies (see Ferguson et al. 1996), a number that is consistent with the $\sim 25 \%$ of ionising photons that are on average missing in the $\mathrm{H}$ II regions with respect to the model predictions. Our study is not much affected by the fraction of ionising photons escaping into the WIM, since our microwave flux estimate from the Cygnus region has been obtained by integrating over a rather larger spatial area, including both individual $\mathrm{H}$ II regions and the WIM (see Sect. 4.1).

Alternatively, ionising photons may be also absorbed by dust within the $\mathrm{H}$ II regions, and a recent study of Inoue et al. (2001) indicates that $\sim 20 \%$ of the photons may be lost in small galactic $\mathrm{H}$ II regions in this way. Using a decomposition of DIRBE far-infrared data, Sodroski et al. (1997) estimated an infrared excess of $1.1-1.7$, corresponding to $5 \%-12 \%$ of Lyc photon absorption within the Galaxy (Mezger et al. 1974). Thus instead of ionising the WIM, the apparent $25 \%$ flux overprediction may be also (partly) explained by dust absorption.

Whatever the mechanism, 25\% ionising flux overprediction by our model should be a rather solid upper limit, which is in any case far from the required factor of 2 that is needed to explain the ${ }^{26} \mathrm{Al}$ nucleosynthesis.

\subsection{Completeness}

In our aim to predict absolute fluxes from the $\mathrm{OB}$ associations and young open clusters in Cygnus, we have to be concerned about the completeness of our database. First, there might be more OB associations in Cygnus than those we listed in Table 1. Due to their extent and weak clustering, OB associations are generally difficult to identify, in particular if they are nearby. To estimate the association completeness, we compared the number of $\mathrm{O}$ stars in the Cygnus area from the SIMBAD database to the number of $\mathrm{O}$ stars in our stellar database. Many entries in the SIMBAD database originate from the $\mathrm{O}$ star catalogue of Garmany et al. (1982), which is complete down to a visible magnitude of $V=10 \mathrm{mag}$, corresponding to a maximum $D M+A_{V}=14.3 \mathrm{mag}$ for O type stars. Comparison of this limit to the distance moduli and reddenings in Table 1 shows that, except for Cyg OB2 and some of the heavily reddened or distant open clusters, the SIMBAD data should indeed be complete for the Cygnus region.

In total we find $171 \mathrm{MK}$ classified $\mathrm{O}$ stars in the surveyed area in the SIMBAD database while our association database contains $204 \mathrm{O}$ stars. Our excess in O stars is due to the 120 objects in the heavily reddened Cyg OB2 asso- ciation that are only partially identified in SIMBAD (about $50 \%$ ). Excluding Cyg OB2 from SIMBAD and our database results in $108 \mathrm{O}$ stars in SIMBAD versus $84 \mathrm{O}$ stars in our database, resulting in a completeness of almost $80 \%$. Adding the $120 \mathrm{O}$ stars of Cyg OB2 to both samples increases the O star completeness to about $90 \%$. Thus, most known $\mathrm{O}$ stars are indeed located in the OB associations of our database.

Secondly, there may be young open clusters in Cygnus that escaped so far detection due to the heavy obscuration in this area. Indeed, using $2 M A S S$ near-infrared data, Dutra \& Bica (2001) found 21 new cluster candidates in our survey region $\left(60^{\circ}<l<110^{\circ}\right)$, which doubles the number of young open clusters in this area. Also, on basis of $2 M A S S$ data, Le Duigou \& Knödlseder (2002) determined the stellar content of 15 new infrared cluster candidates in the area, and concluded that they triple the OB population with respect to the known open clusters in the centre of the Cygnus $\mathrm{X}$ region. However, compared to the giant association Cyg OB2, they contribute only a small fraction, and we find only an additional $10 \%$ of OB stars in the newly discovered clusters candidates.

Thus, in summary, we believe that our database is fairly complete, within about $10-20 \%$, and that the $1.809 \mathrm{MeV}$ luminosity puzzle can not be explained by a hidden population of massive stars. In any case, if there would be such a hidden population, it should also produce a considerable amount of ionising photons, which would lead quickly to an overproduction with respect to the microwave observations.

\subsection{Rotation}

Throughout this work we have ignored the effects of stellar rotation, mainly because there is not sufficient stellar data available for the stellar associations we are interested in, and since there are no stellar grids available so far that allow for inclusion of rotation in evolutionary synthesis calculations. Rotation firstly affects the colour and luminosity of a star, hence it may introduce an artificial age spread in the cluster HRDs (e.g. de Geus 1990; Lamers et al. 1997). Since we accounted for age uncertainties in our approach, however, we do not believe that neglecting of rotation in the determination of the cluster parameters plays an important role.

Much more important may be the impact of rotation on stellar evolution and in particular the nucleosynthesis of ${ }^{26} \mathrm{Al}$. To illustrate this point, we show in Fig. 6 the evolution of the surface ${ }^{26} \mathrm{Al}$ abundance as a function of time for two $60 M_{\odot}$ stellar models at solar metallicity with and without rotation (Ringger 2000). The models were computed with the same physical ingredients as used in Meynet \& Maeder (2000). Let us note in particular that the mass-loss rates used by Ringger (2000) are smaller by a factor of 2-3 than the mass-loss rates used in the stellar models that have been applied to calculate the ${ }^{26} \mathrm{Al}$ yields in this work.

In the case of the rotating models, surface enrichments begin to occur at a much earlier stage than in the non-rotating model (see Fig. 6). Indeed rotational mixing brings freshly synthesised ${ }^{26} \mathrm{Al}$ at the surface well before shells, having experienced $\mathrm{CNO}$ processing, are uncovered by the stellar winds. 


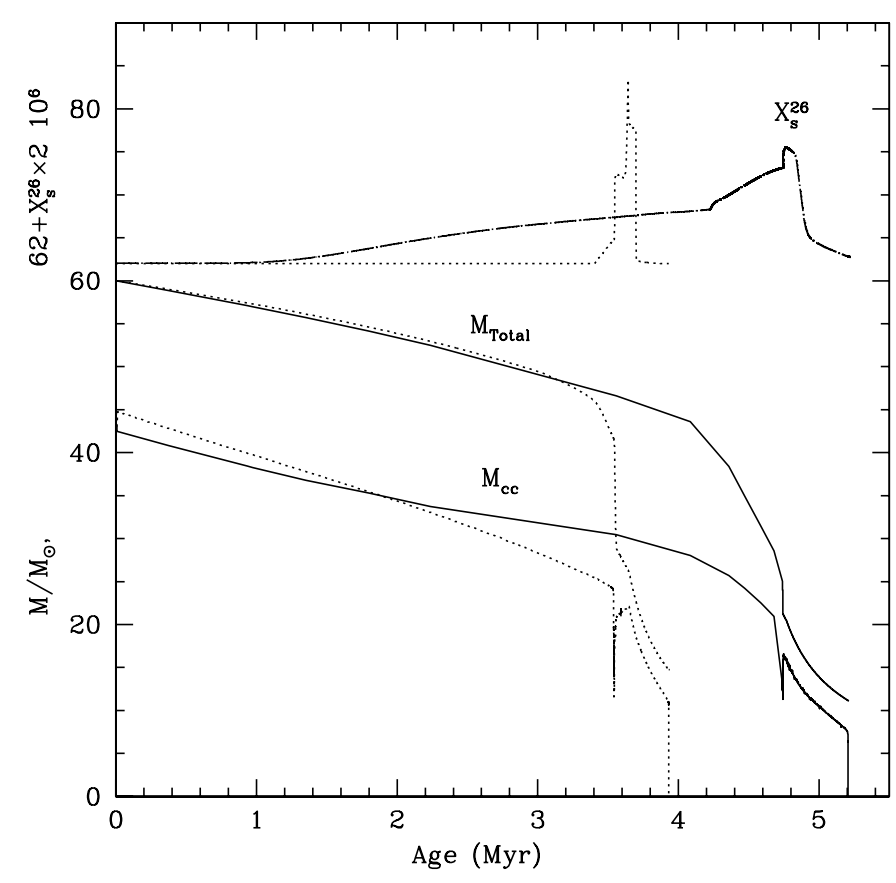

Fig. 6. Evolution as a function of time of the total mass of the star, $M_{\text {Total }}$, of the mass of the convective core, $M_{\mathrm{cc}}$ and of the surface abundance of ${ }^{26} \mathrm{Al}$ in mass fraction, $\mathrm{X}_{\mathrm{s}}^{26}$, for a rotating (continuous lines) and a non-rotating (dotted lines) $60 M_{\odot}$ stellar model at solar metallicity. The initial equatorial velocity for the rotating model is $300 \mathrm{~km} \mathrm{~s}^{-1}$.

This explains why the ${ }^{26} \mathrm{Al}$ abundance at the surface of the rotating model increases in a much smoother way than at the surface of the non-rotating model. One can see also that rotational mixing slows down the decrease in mass of the convective core and increases the Main Sequence lifetime. This results from the diffusion of hydrogen into the convective core. Some ${ }^{25} \mathrm{Mg}$ will also migrate from the radiative envelope into the convective core where it will be transformed into ${ }^{26} \mathrm{Al}$. These effects tend to increase the quantity of ${ }^{26} \mathrm{Al}$ ejected by the stellar winds.

Figure 7 shows the evolution of $M^{26}$, the integrated mass of ${ }^{26} \mathrm{Al}$ ejected by the stellar winds by the rotating and non-rotating $60 M_{\odot}$ models. This quantity is obtained by estimating the integral

$M^{26}(t)=\int_{0}^{t} X_{s}^{26}\left(t^{\prime}\right) \dot{M}\left(t^{\prime}\right) \mathrm{d} t^{\prime}$,

where $\dot{M}$ is the mass loss rate. The luminosity at $1.809 \mathrm{MeV}$ is, at each time, proportional to the mass of non-decayed ${ }^{26} \mathrm{Al}$ (see the dotted lines in Fig. 7). One sees that the maximum luminosity reached by the rotating model is shifted towards later time and is only slightly increased with respect to the non-rotating model. This occurs because in the rotating model, the release of the ${ }^{26} \mathrm{Al}$ is distributed over a much longer time, and therefore, the radionuclide has more time to decay. Thus even if the quantity of ${ }^{26} \mathrm{Al}$ released by the rotating model is significantly greater (for this particular model the enhancement factor is $\sim 1.6$ ), the enhancement of the luminosity is not as great. The above examples show that rotation, all other physical ingredients being kept equal, tends to increase the total quantity of ${ }^{26} \mathrm{Al}$

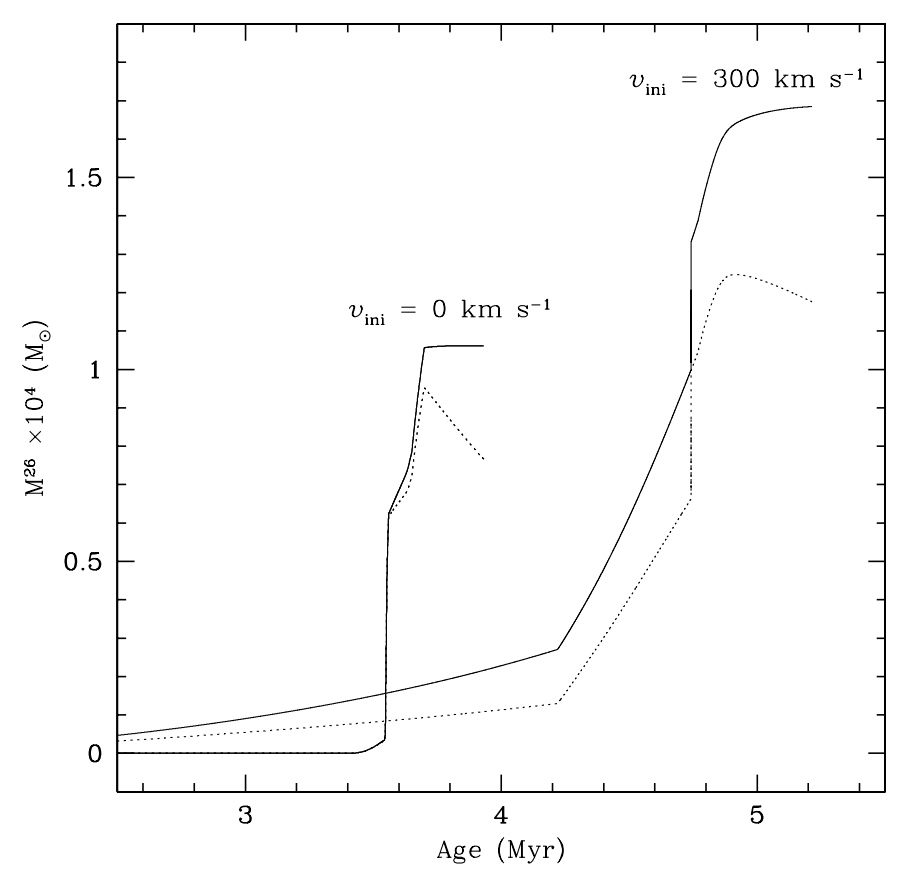

Fig. 7. Continuous lines show the evolution as a function of time of the integrated mass of ${ }^{26} \mathrm{Al}$ ejected by stellar winds for a non-rotating and a rotating $60 M_{\odot}$ stellar model at solar metallicity. The initial equatorial velocities are indicated. The dotted lines show the evolution of the mass of non-decayed ${ }^{26} \mathrm{Al}$.

ejected, slightly increases the maximum $1.809 \mathrm{MeV}$ luminosity, and shifts the ejection of the bulk of ${ }^{26} \mathrm{Al}$ to later times.

How the results of the present population synthesis models would be affected by rotation? First of all, let us recall that the WR stellar models used in the present population synthesis models implicitly account in some way for non-standard physical mechanisms. Indeed these models were computed with artificially enhanced mass loss rates which enabled to account in a satisfactorily manner for the variation with the metallicity of the number of WR to O-type stars (Maeder \& Meynet 1994). Thus the reproduction of this observational constraints in some way calibrates these stellar models. If we compare the total quantity of ${ }^{26} \mathrm{Al}$ ejected by our rotating $60 M_{\odot}$ model with the non-rotating $60 M_{\odot}$ model with enhanced mass loss rates, used in the present population synthesis model, we find a modest ${ }^{26} \mathrm{Al}$ yield increase of $13 \%$.

Does this mean that rotation has to be discarded as a possible mechanism for solving the ${ }^{26} \mathrm{Al}$ deficit discussed in the present paper? We think it is too early to reach such a conclusion. Since the work of Ringger (2000), new improvements of the physics of rotation have been achieved especially concerning the shear diffusion coefficient and the impact of rotation on the mass loss rates (Maeder \& Meynet 2000, 2001). Predictions of precise enhancement factors requires the computation of stellar models for different initial masses and rotational velocities. At the present time, no such grids of models, predicting the $1.809 \mathrm{MeV}$ luminosity, have been computed. We therefore defer a more quantitatively assessment of rotation on gamma-ray line emission in Cygnus to future work. 


\subsection{Binarity}

Binarity may be another mechanism that could alter the ${ }^{26} \mathrm{Al}$ yields from massive stars, yet we did not account for their effects due to the involved complexity and poorly known details. In particular, only close binary systems are concerned, and there is not sufficient data available for the associations we are interested in to assess their number and mass distribution.

Two effects may impact the nucleosynthesis in close binary systems: tidal interactions in close binary systems, and mass transfer by Roche Lobe overflow. Tidal effects are expected to deform the star and therefore induce instabilities reminiscent of those induced by rotation. In particular, tidal forces and orbital motions will induce rotation even in initially non-rotating stars. To our knowledge, this effect has never been studied in nucleosynthesis calculations, despite its potentially important consequences, as for instance by homogenising the stars.

If mass transfer occurs, the removal of part of the envelope of the donor star may favour the appearance of ${ }^{26} \mathrm{Al}$ on the surface, in a similar fashion as mass-loss acts through stellar winds. More important changes may occur for the gainer in systems with $M<40 M_{\odot}$, as e.g. shown by the preliminary studies of Braun \& Langer (1995) and Langer et al. (1998). The latter suggest a scenario in which mass transfer onto the secondary leads to a rejuvenation which alters its subsequent evolution. They predict an increase by $2-3$ orders of magnitudes of the hydrostatically produced ${ }^{26} \mathrm{Al}$ yield due to a reduction of the delay between production and ejection of ${ }^{26} \mathrm{Al}$, which may possibly enhance the total ${ }^{26} \mathrm{Al}$ production from Type II supernovae by a factor of about 2 (Langer, priv. communication). Since, however, supernovae are very rare in Cygnus, it is highly doubtful that such a mechanism could solve the ${ }^{26} \mathrm{Al}$ yield puzzle.

\section{Conclusions}

\subsection{Nucleosynthesis}

Our modelling effort of the gamma-ray line emission from $\mathrm{OB}$ associations and young open clusters in the Cygnus region has revealed a possible shortcoming of actual nucleosynthesis models in explaining ${ }^{26} \mathrm{Al}$ production. We find a $1.809 \mathrm{MeV}$ flux underestimation of about a factor of 2 that is difficult to explain by other means than a modification of current nucleosynthesis models for single, non-rotating stars. From preliminary calculations, it appears difficult for rotation alone to significantly enhance ${ }^{26} \mathrm{Al}$ production, although this conclusion needs to be checked by more detailed calculations in the future.

Only little ${ }^{60} \mathrm{Fe}$ production is predicted in Cygnus by our model, mainly related to the low number of recent supernova events in this region. A detection of the $1.137 \mathrm{MeV}$ and $1.332 \mathrm{MeV}$ lines from the radioactive decay of ${ }^{60} \mathrm{Fe}$ by INTEGRAL would therefore present a big surprise. In the case of such a detection, ${ }^{60} \mathrm{Fe}$ production by hydrostatic helium burning in Wolf-Rayet stars - which we have not included in our model due to the low yield predictions - should then be seriously reconsidered (Arnould et al. 1997).

\subsection{Other gamma-ray signatures}

In addition to ${ }^{26} \mathrm{Al}$ and ${ }^{60} \mathrm{Fe}$, massive star associations may produce further radioactive isotopes during the supernova explosion of massive stars, such as ${ }^{56} \mathrm{Co}$ and ${ }^{44} \mathrm{Ti}$, yet their short lifetimes of 112 days and 87 years, respectively, make their observation impossible in absence of a very recent event. However, ${ }^{56} \mathrm{Co}$ and ${ }^{44} \mathrm{Ti}$ decay under positron emission (like ${ }^{26} \mathrm{Al}$ ), and the annihilation of positrons with electrons of the interstellar medium on time scales of a few $10^{5}$ years may provide a reverberation of the short-lived, extinct, radioactivities. Hence, the observation of the $511 \mathrm{keV}$ positron annihilation line may provide an independent measure of the supernova activity in massive star associations (and in particular in Cygnus), although the interpretation of the observations will be complicated by the annihilation physics, the positron transport, and the positron escape fraction from the expanding supernova remnants.

\subsection{Super star clusters}

Gamma-ray as well as free-free emission in the Cygnus region seems to be dominated by a single, extremely massive association: Cyg OB2. Indeed, Cyg OB2 is merely a prototype of a young globular cluster than an OB association (Knödlseder 2000). There are examples of further super star clusters in the Galaxy, such as NGC 3603 (Moffat et al. 1994), the Arches and Quintuplet clusters near the galactic centre (Figer et al. 1999), or the W49A cluster (Conti \& Blum 2002). Most of these super star clusters are partially or totally obscured in the visible by the absorbing effects of intervening and/or local interstellar dust, and it is unclear how many of them exist throughout the entire Galaxy. Making the simplifying assumption that super star clusters are distributed uniformly throughout the Galaxy within a galactocentric distance of $15 \mathrm{kpc}$, and taking that Cyg OB2 is apparently the only such object within $1.5 \mathrm{kpc}$, one may expect 100 super star clusters in our Galaxy. Assuming that they all produce $10^{-2} M_{\odot}$ of ${ }^{26} \mathrm{Al}$, similar to our Cyg OB2 model prediction, a total ${ }^{26} \mathrm{Al}$ production of $1 M_{\odot}$ is expected from these objects. Taking into account the ${ }^{26} \mathrm{Al}$ yield underestimation of a factor of 2 brings this mass to $2 M_{\odot}$, comparable to the observed galactic ${ }^{26} \mathrm{Al}$ mass of 2-3 $M_{\odot}$ (Diehl et al. 1995; Knödlseder 1999). Thus, a considerable fraction of ${ }^{26} \mathrm{Al}$ may indeed be produced by such super star clusters, and $1.809 \mathrm{MeV}$ gamma-ray observations with sufficient angular resolution and sensitivity may help to uncover and to study them throughout the Milky-Way.

\subsection{The Cygnus superbubble}

Our age determination of clusters in the Cygnus region sheds some doubt on the triggered star formation scenario that has been proposed to explain anomalous stellar proper motions in the area (Comerón \& Torra 1994). In this scenario, the central association Cyg OB2 is supposed of having formed a shell blown by stellar winds and supernovae, the Cygnus superbubble, that subsequently gave birth to the surrounding associations Cyg OB1, OB3, OB7 and OB9 due to gravitational shell 
instability. However, our age estimate for Cyg OB2 is inferior to that of the surrounding associations, making triggered star formation in this area unlikely. In contrast, the anomalous stellar proper motions are equally well explained by supposing that they result from dynamically ejected runaway stars from Cyg OB2 (Comerón et al. 1998), in particular since the proposed expansion age of $\sim 4 \mathrm{Myr}$ is compatible with our age estimate for Cyg OB2.

\subsection{Cygnus and the Galaxy}

Finally, we want to mention that not only the Cygnus region but also the Galaxy as a whole suffers probably from an underproduction of ${ }^{26} \mathrm{Al}$ when current, non-rotating nucleosynthesis models are considered. By combining the same nucleosynthesis models that have been used in this work with the atmosphere models of Schaerer \& de Koter (1997), and normalisation to the observed galactic Lyman continuum luminosity, Knödlseder (1999) predicted a galactic ${ }^{26} \mathrm{Al}$ mass of $1.6 M_{\odot}$, significantly below the observed value of 2-3 $M_{\odot}$. Only the inclusion of the galactic metallicity gradient, which considerably enhances ${ }^{26} \mathrm{Al}$ ejection through stellar winds in the Wolf-Rayet phase towards the galactic centre, provides a sufficient increase of the galactic ${ }^{26} \mathrm{Al}$ production, bringing the model predictions in better agreement with the observations.

Yet, metallicity is of no help in the Cygnus region, where even slightly subsolar abundances are reported (Daflon et al. 2001). On the other hand, if another process, such as rotation, is needed to explain the ${ }^{26} \mathrm{Al}$ production in Cygnus, it should also be at work for the entire Galaxy, leading to a potential ${ }^{26} \mathrm{Al}$ overproduction if combined with the metallicity enhancement. In fact, if ${ }^{26} \mathrm{Al}$ production by massive stars would indeed follow the $\left(Z / Z_{\odot}\right)^{2}$ dependence predicted by non-rotating stellar models, a break-down of the tight correlation between $1.809 \mathrm{MeV}$ and galactic free-free emission would be expected due to the inverse metallicity dependencies of both emission processes the $1.809 \mathrm{MeV}$ intensity should be enhanced in the metal-rich inner regions of the Galaxy due to enhanced ${ }^{26} \mathrm{Al}$ production, while the free-free emission should be reduced due to lower electron temperatures (see Knödlseder 1999).

The existing data show no indication for such an anticorrelation. However, it is likely that rotation reduces the metallicity dependence of Wolf-Rayet star yields. The fact that at low metallicity, there is less ${ }^{25} \mathrm{Mg}$, might be somewhat compensated by the fact that when the metallicity decreases, the mixing is more efficient, bringing more ${ }^{25} \mathrm{Mg}$ from the radiative envelope into the core and more ${ }^{26} \mathrm{Al}$ from the convective core into the radiative envelope. The mixing efficiency increases when the metallicity decreases because, when $Z$ decreases, the stars are more compact, the internal gradients of the angular velocity are steeper and the stars loose less angular momentum by mass loss through stellar winds (see Maeder \& Meynet 2001). Secondly, when rotation is accounted for, the mass loss rate plays a less important role in the WR formation process (see Fig. 6). Thirdly, at low metallicity, stars loose less angular momentum. As a consequence they can more easily reach the break-up limit at a given stage during their evolution. Very high mass loss rates ensue even if the metallicity is low. Finally, there are some indirect indications, that the proportion of fast rotators increases when the metallicity decreases (Maeder et al. 1999).

Since rotation seems to erode the metallicity dependence of Wolf-Rayet star yields, it provides an appealing mechanism to explain the absence of a $1.809 \mathrm{MeV}$ - free-free emission anti-correlation. Remains to be seen if rotation can also be a solution to the ${ }^{26} \mathrm{Al}$ yield puzzle - both for the Cygnus region and the Galaxy as a whole.

Acknowledgements. This research has made use of the SIMBAD database, operated at CDS, Strasbourg, France and the WEBDA database, compiled by Jean-Claude Mermilliod, Institute of Astronomy of the University of Lausanne, Switzerland.

\section{References}

Afflerbach, A., Churchwell, E., \& Werner, M. W. 1997, ApJ, 478, 190 Arnould, M., Paulus, G., \& Meynet, G. 1997, A\&A, 321, 452

Balog, Z., Delgado, A. J., Moitinho, A., et al. 2001, MNRAS, 323, 872

Baumgardt, H. 1998, A\&A, 340, 402

Bennett, C. L., Smoot, G. F., Hinshaw, G., et al. 1992, ApJ, 396, L7

Blaha, C., \& Humphreys, R. M. 1989, AJ, 98, 1598

Bochkarev, N. G., \& Sitnik, T. G. 1985, Ap\&SS, 108, 237

Braun, H., \& Langer, N. 1995, A\&A, 297, 483

Cash, W., Garmire, G., \& Riegler, G. 1980, ApJ, 238, L71

Cerviño, M., Knödlseder, J., Schaerer, D., von Ballmoos, P., \& Meynet, G. 2000, A\&A, 363, 970

Clayton, G. C., \& Fitzpatrick, E. L. 1987, AJ, 92, 157

Comerón, F., Torra, J., Jordi, C., \& Gómez, A. E. 1993, A\&AS, 101, 37

Comerón, F., \& Torra, J. 1994, ApJ, 423, 652

Comerón, F., Torra, J., \& Gómez, A. E. 1998, A\&A, 330, 975

Conti, P. S., \& Blum, R. D. 2002, ApJ, 564, 827

Daflon, S., Cunha, K., Becker, S. R., \& Smith, V. V. 2001, ApJ, 552, 309

Deeg, H. J., \& Ninkov, Z. 1996, A\&AS, 119, 221

de Jager, C., Nieuwenhuijzen, H., \& van der Hucht, K. A. 1988, A\&AS, 72, 259

Del Rio, E., von Ballmoos, P., Bennett, K., et al. 1996, A\&A, 315, 237

De Geus, E. J. 1990, in Properties of hot luminous stars; Proceedings of the First Boulder-Munich Workshop, Boulder, CO, Aug. 6-11, 1988 (A90-36851 15-90) (San Francisco, CA, Astronomical Society of the Pacific), 3

De Zeeuw, P. T., Hoogerwerf, R., de Bruijne, J. H. J., Brown, A. G. A., \& Blaauw, A. 1999, AJ, 117, 354

Diehl, R., Dupraz, C., Bennett, K., et al. 1995, A\&A, 298, 445

Dupuy, D. L., \& Zukauskas, W. 1976, J. Royal Astr. Soc. Canada, 70, 169

Dutra, C. M., \& Bica, E. 2001, A\&A, 376, 434

Ferguson, A. M. N., Wyse, R. F. G., Gallagher, J. S., \& Hunter, D. A. 1996, AJ, 111, 2265

Figer, D. F., Kim, S. S., Morris, M., et al. 1999, ApJ, 525, 750

FitzGerald, M. P. 1970, A\&A, 4, 234

Garmany, C. D. 1994, PASP, 106, 25

Garmany, C. D., \& Stencel, R. E. 1992, A\&AS, 94, 211

Garmany, C. D., Conti, P. S., \& Chiosi, C. 1982, 263, 777

Garrison, R. F., \& Kormendy, J. 1976, PASP, 88, 865

Herrero, A., Corral, L. J., Villamariz, M. R., \& Martin, E. L. 1999, A\&A, 348, 542 
Hoogerwerf, R., Bruijne, J. H. J., \& de Zeeuw, P. T. 2001, A\&A, 365,49

Huchtmeier, W. K., \& Wendker, H. J. 1977, A\&A, 58, 197

Humphreys, R. M., \& McElroy, D. B. 1984, ApJ, 284, 565

Humphreys, R. M. 1978, ApJS, 38, 309

Hunter, D. A., \& Massey, P. 1990, AJ, 99, 846

Inoue, A. K., Hirashita, H., \& Kamaya, H. 2001, 555, 613

Knödlseder, J., \& Vedrenne, G. 2001, in Exploring the Gamma-Ray Universe, ed. A. Gimenez, V. Reglero, \& C. Winkler, 23

Knödlseder, J. 2000, A\&A, 360, 539

Knödlseder, J., Bennett, K., Bloemen, H., et al. 1999a, A\&A, 344, 68

Knödlseder, J., Dixon, D., Bennett, K., et al. 1999b, A\&A, 345, 813

Knödlseder, J. 1999, ApJ, 510, 915

Lamers, H. J. G. L. M., Harzevoort, J. M. A. G., Schrijver, H., et al. 1997, A\&A, 325, L25

Lamers, H., \& Cassinelli, J. P. 1996, Mass Loss from Stars, in From Stars to Galaxies: The Impact of Stellar Physics on Galaxy Evolution, ed. C. Leitherer, U. Fritze-von-Alvensleben, \& J. Huchra, ASP Conf. Ser., 98, 162

Langer, N. 1998, A\&A, 329, 551

Langer, N., Braun, H., \& Wellenstein, S. 1998, in Proceedings of the 9th Workshop on Nuclear Astrophysics, ed. W. Hillebrandt, \& E. Müller, 18

Le Duigou, J. M., \& Knödlseder, J. 2002, A\&A, submitted

Maeder, A., \& Meynet, G. 2001, A\&A, 373, 555

Maeder, A., \& Meynet, G. 2000, A\&A, 361, 159

Maeder, A., Grebel, E., \& Mermilliod, J. C. 1999, A\&A 346, 459

Maeder, A., \& Meynet, G. 1994, A\&A, 287, 803

Maeder, A. 1992, A\&A, 264, 105

Marschall, L. A., Comins, N. F., \& Karshner, G. B. 1990, AJ, 99, 1536

Martins, F., Schaerer, D., \& Hillier, D. J. 2002, A\&A, 382, 999

Massey, P., DeGioia-Eastwood, K., \& Waterhouse, E. 2001, AJ, 121, 1050

Massey, P., Johnson, K. E., \& DeGioia-Eastwood, K., et al. 1995, ApJ, 454, 151

Massey, P., \& Thompson, A. B. 1991, AJ, 101, 1408

Mathis, J. S. 1990, ARA\&A, 28, 37

Mermilliod, J.-C. 1998, Bull. Inform., CDS 35, 77 http://obswww. unige.ch/webda/webda.html

Meynet G., \& Maeder A. 2000, A\&A, 361, 101

Meynet, G., Arnould, M., Prantzos, N., \& Paulus, G. 1997, A\&A, 320, 460
Meynet, G., Maeder, A., Schaller, G., Schaerer, D., \& Charbonnel, C. 1994, A\&AS, 103, 97

Mezger, P. G., Smith, L. F., \& Churchwell, E. 1974, A\&A, 32, 269

Moffat, A. F. J., Drissen, L., \& Shara, M. M. 1994, ApJ, 436, 183

Moffat, A. F. J. 1971, A\&A, 13, 30

Morbidelli, L., Patriarchi, P., Perinotto, M., Barbaro, G., \& di Bartolomeo, A. 1997, A\&A, 327, 125

Oey, M. S., \& Kennicutt, R. C. Jr. 1998, PASA, 15, 141

Oey, M. S., \& Kennicutt, R. C. Jr. 1997, MNRAS, 291, 827

Panagia, N. 1973, AJ, 78, 929

Parthasarathy, M., Jain, S. K., \& Bhatt, H. C. 1992, A\&A, 266, 202

Pigulski, A., \& Kolaczkowski, Z. 1998, MNRAS, 298, 753

Plüschke, S. 2001, Ph.D. Thesis, MPE

Prantzos, N., \& Diehl, R. 1996, Phys. Rep., 267, 1

Racine, R. 1969, AJ, 74, 816

Ringger, D. 2000, Diploma work, Lausanne University

Robichon, N., Arenou, F., Mermilliod, J.-C., \& Turon, C. 1999, A\&A, 345,471

Schaerer, D. 1999, in Stars, Gas and Dust in Galaxies: Exploring the Links, ed. D. Alloin, G. Galaz, \& K. Olsen, ASP Conf. Ser., 221, 99

Schaerer, D. 1999, in Boulder Munich Workshop II: Properties of Hot Luminous Stars, ed. I. Howarth, ASP Conf. Ser., 131, 310

Schaerer, D., \& de Koter 1997, A\&A, 322, 598

Schmidt-Kaler, T. 1982, Landolt-Börnstein, VI, 2, 453

Schmutz, W., Leitherer, C., \& Gruenwald, R. 1992, PASP, 104, 1164

Sodroski, T. J., Odegard, N., Arendt, R. G., et al. 1997, ApJ, 480, 173

Torres-Dodgen, A. V., Tapia, M., \& Carroll, M. 1991, MNRAS, 249, 1

Turner, D. G. 1998, AJ, 118, 274

Turner, D. G., \& Forbes, D. 1982, PASP, 94, 789

Uyaniker, B., Fürst, E., Reich, W., Aschenbach, B., \& Wielebinski, R. 2001, A\&A, 371, 675

Vacca, W. D., Garmany, C. D., \& Shull, J. M. 1996, ApJ, 460, 914

van der Hucht, K. A. 2001, New Astr. Rev., 45, 135

Wang, J.-J., \& Hu, J.-Y. 2000, A\&A, 356, 118

Wendker, H. J., Higgs, L. A., \& Landecker, T. L. 1991, A\&A, 241, 551

Wendker, H. J. 1970, A\&A, 4, 378

Wilking, B. A., Harvey, P. M., \& Joy, M. 1984, AJ, 89, 496

Woosley, S., \& Weaver, T. A. 1995, ApJS, 101, 181

Woosley, S., Langer, N., \& Weaver, T. A. 1995, ApJ, 448, 315

Zakirov, M. M. 1999, Astron. Lett., 25, 229 